Physics Vol. 1, No. 5, pp. 279-305, 1965. Physics Publishing Co. Printed in Great Britain

\title{
MAGNETIC RESONANCE OF HIGH QUALITY FERROMAGNETIC METAL SINGLE CRYSTALS*
}

\author{
D. S. RODBELL \\ General Electric Research Laboratory, \\ Schenectady, N.Y., U.S.A.** \\ (Received 26 January 1965)
}

\begin{abstract}
This paper describes the observations and associated analyses of the microwave resonance absorption of very small, apparently highly perfect, single crystal samples of ferromagnetic metals. The primary focus of this study is on nickel but iron and cobalt are also considered. From the point of view of magnetic resonance, the observed narrow line-widths of these samples suggests that they are of high quality compared to bulk material. The general conclusions drawn are that the limited penetration of the microwave energy into a conducting ferromagnet makes an important contribution to the observed resonance absorption linewidth and shape. A damping term of the Landau-Lifshitz form is also identified and gives, quite accurately, the observed linewidth and shape behaviour both in temperature and frequency; this without an explicit temperature or frequency dependence of the damping 'constant'. There is no evidence for surface spin pinning being present and, in fact, strong experimental evidence against it playing a role in these studies is presented. The magnetocrystalline anisotropy constants and the $g$ value of nickel metal are deduced from the data. Both $K_{1}$ and $K_{2}$ are found to be negative over the entire range studied $\left(130^{\circ}\right.$ to $\left.635^{\circ} \mathrm{K}\right)$ and both have rapidly decreasing magnitudes with increasing temperature. The spectroscopic splitting factor, $\mathrm{g}$, is determined to be $2.22 \pm .03$ and is independent of temperature and frequency.
\end{abstract}

\section{Introduction}

IN A PERCEPTIVE and anticipatory study of the 'dispersion of magnetic permeability' in solids, Landau and Lifshitz [1], in 1935, described almost the entire range of classical magnetic properties that have since been (and are still being) observed. The discovery by Griffiths [2], in 1946, of ferromagnetic resonance was the first observation of one of the behaviors of a ferromanetic solid considered

* Preliminary accounts of this work have appeared as Phys. Rev. Letters 13, 471 (1964), and in the Proceedings of the International Conference on Magnetism (Nottingham 1964) to be published.

* This paper was written at the clarendon Laboratory, Oxford University, during part of the tenure of a John Simon Guggenheim Memorial Fellowship. 
by Landau and Lifshitz; though to be sure, they had considered it in broad outline only. It is a curious fact that although Griffiths' discovery was made on samples of iron, cobalt and nickel, these elemental ferromagnetic metals have stood in the background of experimental efforts to pursue and understand the detailed behavior of ferromagnetic resonance in solids. The main reason for this is the large breadth of the resonance absorption, characteristic of bulk metallic samples (including monocrystals), compared to that of the insulating magnetic solids. It seems clear from the work thus far that the linewidth is in some way related to the structural perfection of the crystal - both on a macroscopic and a microscopic scale. Macroscopic imperfections must give rise to inhomogeneities in dipolar fields while the microscopic imperfections give rise to aperiodicities that scatter spin waves - or viewed otherwise - give rise to fluctuations in exchange fields. In addition, the strain fields associated with many kinds of imperfections give rise to fluctuations in the magnetocrystalline anisotropy energy and hence contribute to additional local fluctuations. There are other line breadth contributions but, in general, one cannot study them with satisfaction in samples whose behavior is already complicated by imperfections. Metallic ferromagnets, when viewed from their resonance absorption linewidth, would seem to be very prone to have imperfections and/or be very sensitive to their presence. No bulk ferromagnetic metals have yet been reported that exhibit linewidths that may be completely accounted for in a satisfactory way. In this paper there are described the observations and associated analysis of the microwave resonance absorption of very small, apparently highly perfect, single crystal samples of ferromagnetic metals. The primary focus will be upon the properties of nickel but we shall also refer to results obtained in iron. The general conclusion of this work is that the effects that result from the limited penetration of the high frequency fields into metallic conductors can account for an important part of the observed behavior in ferromagnetic metals but, additionally, there is strong evidence for the existence of an intrinsic damping term that has precisely the functional form assumed by Landau and Lifshitz in their analysis. The functional form of this term may be obtained readily from a simple geometrical argument that expands $d \bar{M} / d t$ into the orthogonal coordinates $\bar{M}, \bar{M} \times \bar{H}$ and $\bar{M} \times[\bar{M} \times \bar{H}]$. When $|\bar{M}|$ is a constant of the motion and $\bar{M} \times \bar{H}$ determines the resonance frequency, the $\bar{M} \times[\bar{M} \times \vec{H}]$ term describes the breadth of the resonance absorption. The coefficient of this term may be determined experimentally but its microscopic origin is presently uninown.

\section{Previous studies}

Before describing the present experimental findings with their interpretation and analysis it seems appropriate to describe some of the salient historical points in this area of physics. This description must of necessity be brief, but it will try to convey the importance of certain historical stepping stones upon which the current work relies.

With the initial observations of ferromagnetic resonance came two major problems; first, the position of the resonance absorption, and second, its breadth.

If the position (the applied external field) about which the resonance is centered, is interpreted naively as the total 'effective field' (i.e. $\omega=\gamma H_{\text {eff }}$ ) then no sense can be made of the observations - not only in metals but in all types of samples (except spherical, isotropic, insulating materials). It was Kittel [3] who pointed out the importance of demagnetizing fields in these experiments. When both the r.f. and static demagnetization factors are considered, then a spectroscopic splitting factor is deduced (using $\omega / \gamma=(B H)^{1 / 2}$ ) from the observations that is characteristic of an electronic spin system, and is consistent for many of the materials examined. This classical analysis was later put on firm quantum mechanical ground by Van vleck [4] and by others. In its classical form it has been generalized (independently) by Suhl [5] and by Smit and Beljers [6] to an often useful formulation which considers the total orientation 
dependent energy, $E(\theta, \varphi)$, in the polar and azimuthal coordinates $(\theta, \phi)$, and shows that in the equilibrium orientation $\left(\frac{\partial E}{\partial \theta}=\frac{\partial E}{\partial \phi}=0\right)$ the effective field of the system so considered may be determined by examining a Taylor expansion of the energy about the equilibrium orientation. The restoring torque may be expressed as originating in the effective field

$$
H_{\text {ef } f}=\frac{1}{M \sin \theta}\left[\left(\frac{\partial^{2} E}{\partial \theta^{2}}\right)\left(\frac{\partial^{2} E}{\partial \phi^{2}}\right)-\left(\frac{\partial^{2} E}{\partial \theta \partial \phi}\right)^{2}\right]^{1 / 2}
$$

Thus the position of the resonance absorption may be used to deduce some of the important contributory sources of $E(\theta, \phi)$ such as the magneto-crystalline anisotropy energy. Strictly speaking, this analysis applies to the 'uniform precession' of all spins and cannot involve exchange

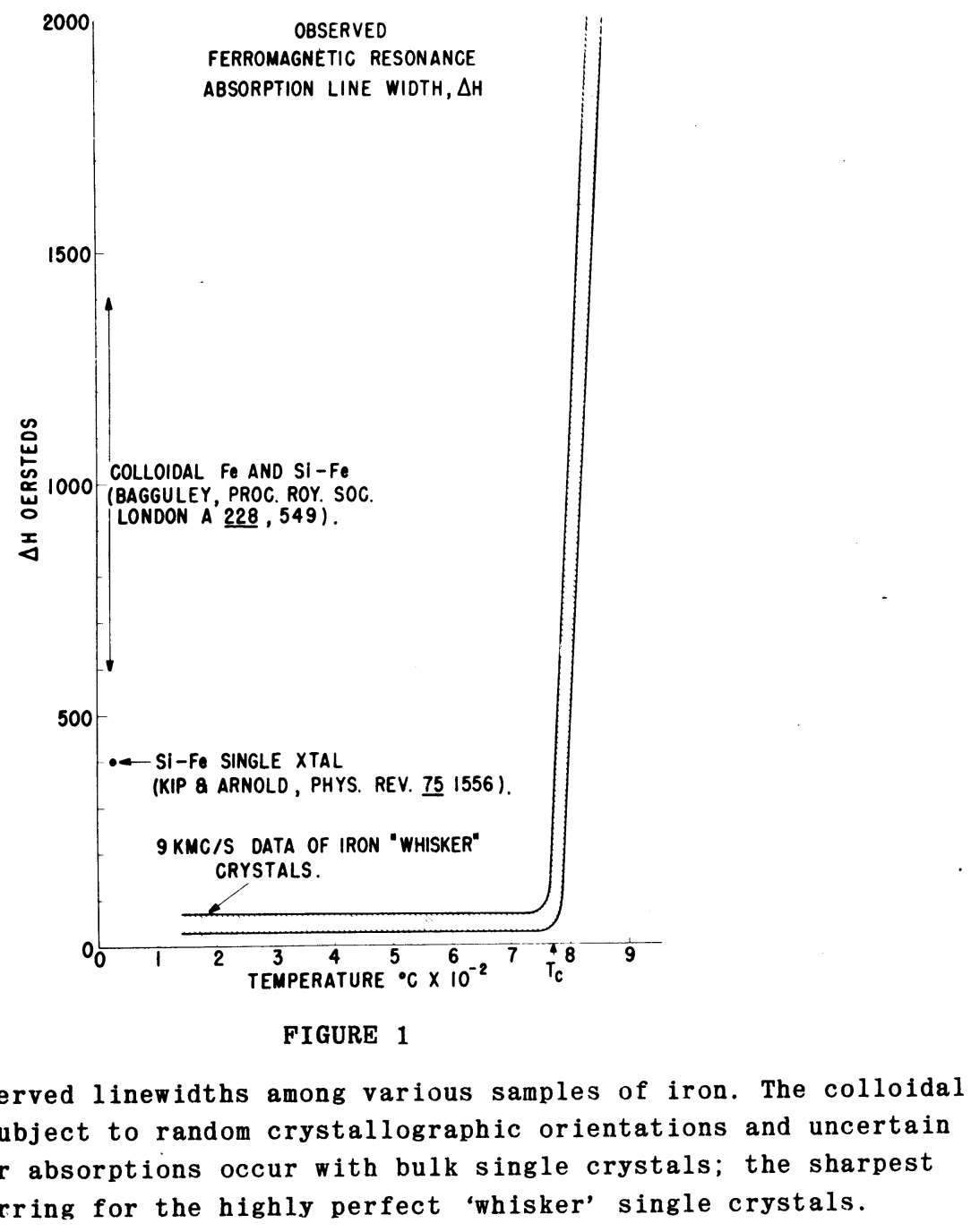

energy unless neighboring spins assume non parallel orientations during their motions. Herring and Kittel [7] considered 'spin waves' in ferromagnets, where exchange energy is non-trivial and subsequently Ament and Rado [8] (and Macdonald [9] independently) did a proper calculation of resonance in metallic ferromagnets, where in fact, the resonance mode excited is not the 
uniform precession but a long wavelength spin wave. Clogston, Suhl, Walker and Anderson [10], in connection with their search for a line width mechanism in insulating ferromagnets, also calculated the spin wave spectrum of an ellipsoidal ferromagnet and Walker [11] calculated the long wavelength excitations (magnetostatic modes); which calculation was extended by Eshback and Damon [12] to include flat plates. The point of this (admittedly incomplete) survey of resonance position calculations is to indicate that the accounting of the observed resonance field is on a firm basis and that we shall develop the analytic tools for our experimental interpretation of that property from existing - almost routine - formulation.

The question of linewidth, as noted in the introduction, has not been on as firm ground in metals as in insulators. Griffiths' published data on nickel indicated a linewidth of about 2000 oersteds (at $9 \mathrm{kMc}$ and $25^{\circ} \mathrm{C}$ ). From experience in paramagnetic resonance one might expect

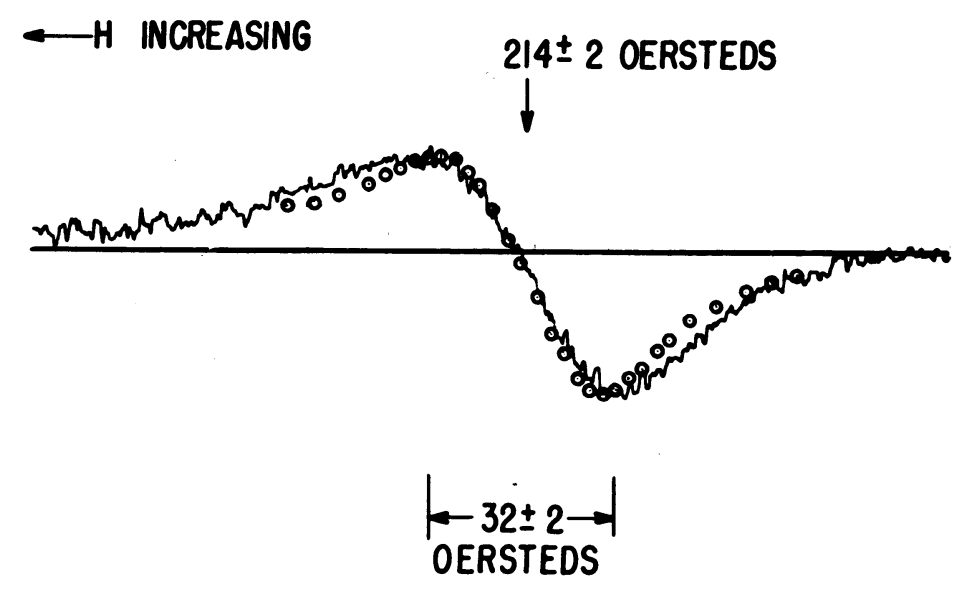

FIGURE 2

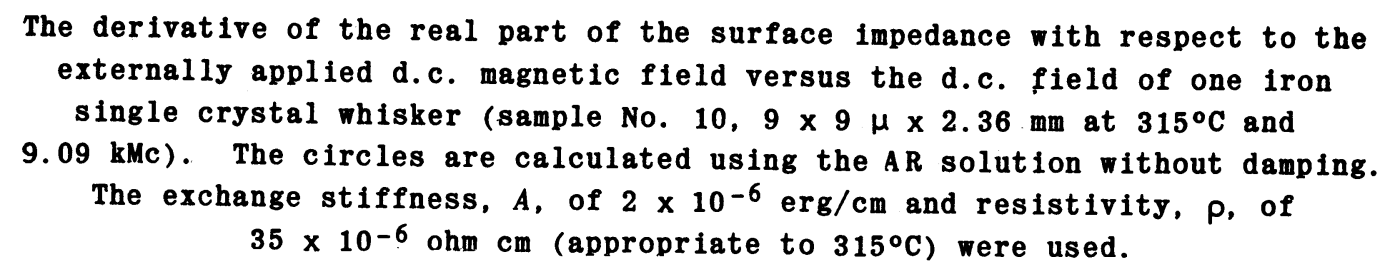

that, in this limiting case of 'exchange narrowing', a very much smaller linewidth - perhaps a few oersteds - would be observed. There are several mitigating circumstances; first of all, polycrystalline samples are subject to an inhomogeneously broadened resonance simply because of the spatial randomness associated with the crystallite orientations relative to the applied field and consequently a breadth of about the 'anisotropy field' is to be expected. Strain and structural imperfections also contribute to the line width. For example the 2000 oersted linewidth of nickel found by Griffiths corresponds to between 500 and 1000 oersteds found in polished bulk single crystal samples $[13,14,15]$. In the present study, with very small 'whiskers' and platelets of nickel, * the linewidth of these apparently highly perfect samples is often less than 150 oersteds. A similar situation is to be found in the behaviour of metallic iron. A randomly oriented group of colloidal iron single crystals gives linewidths of about * These samples were grown by Dr. R.W. De Blois of the General Electric Research Laboratory,
Schenectady, N.Y. 
1000 oersted [16]; a bulk single crystal of $3 \%$ Silicon-iron showed a 400 oersted [17] linewidth. Other silicon-iron crystals, carefully handled and polished, have linewidths near 50 oersteds [15]. Selected iron-whisker crystals show linewidths often less than 50 oersteds [18]. These facts are compared graphically in Fig. 1. There also exists some evidence that highly perfect crystals have relatively narrow linewidths in the case of cobalt metal [19]. Taken together, these data are strong support for the position that line width is related to perfection, a position that rests to some extent on theoretical grounds and was sketched briefly in the introduction. In earlier work on iron whiskers it was shown [18] that, without damping, the Ament-Rado formulation of the electromagnetic boundary value problem for metallic ferromagnets was consistent with observations in an appreciable range of temperature near room temperature, but left as unanswered the origin of the apparent constancy of the high temperature line widths (until close to the Curie temperature). From the present vantage point - having studied and analysed the behaviour of nickel whiskers - it seems that, in fact, an additional line width contribution (of the Landau-Lifshitz form) is appropriate to describe that behaviour of iron 'whisker' crystals. The observations on nickel, as we shall soon see, are not only consistent with that interpretation but do indeed require a damping term of precisely that form. Let us now turn our attention to the observed resonance behaviour to see the evidence upon which these statements are based.

\section{Observations, Analysis and Discussion}

The samples of interest in these studies were all grown and supplied by Dr. R.W. De Blois of the General Electric Research Laboratory. The growth of these metallic filaments and platelets was accomplished by the hydrogen reduction of a halide salt of the metal (iron, nickel or cobalt). The bromide salt is used frequently although it is not unique in its ability to be

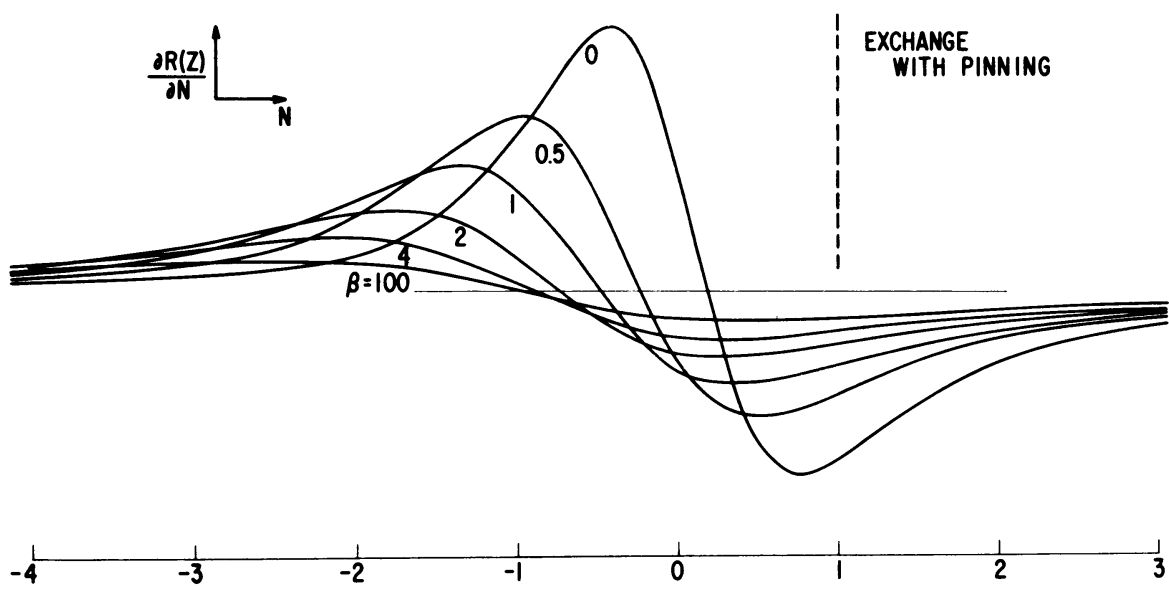

FIGURE 3

The effect of 'pinning' on the exchange-dominated resonance absorption line shape. The derivative of the real part of the surface impedance with respect to field is shown for values of the 'strength' of the pinning ranging from 0 to $\infty$.

The parameter $\beta$ that characterizes the pinning strength is defined in the text. For nickel, $\beta=1$ corresponds to a 'surface anisotropy' of about $1 \mathrm{erg} / \mathrm{cm}^{2}$. The dashed line indicates where the resonance would occur (as a $\delta$-function) in the absence of the exchange shift and broadening here considered. 
reduced to metal single crystals. The question of filamentary or platelike growth habit (and the associated crystallography) seems to be a delicate one and depends upon temperature, rate of hydrogen flow and the dilution of hydrogen with inert gases. Suffice it to say, that although one cannot always predict the results of a given growth attempt, at least the resultant crystals can be selected and identified with standard X-ray techniques and their magnetic resonance properties determined. In size these samples have a typical maximum dimension of a millimeter. For instance, a nickel platelet, might be a millimeter on a side and a few microns thick while a nlckel whisker might typically be a millimeter in length with a cross section of $2 \times 2$ microns. When carefully selected for optical qualities (i.e. specular surfaces and no gross imperfections) these samples often give narrow resonance absorption linewidths. In an earlier study we showed that the observed linewidth of iron whisker crystals can often be found in close correspondence to that predicted to result in metals (without reliance on intrinsic damping) by Ament and Rado. The prediction is based on the solution of the propagation of electromagnetic

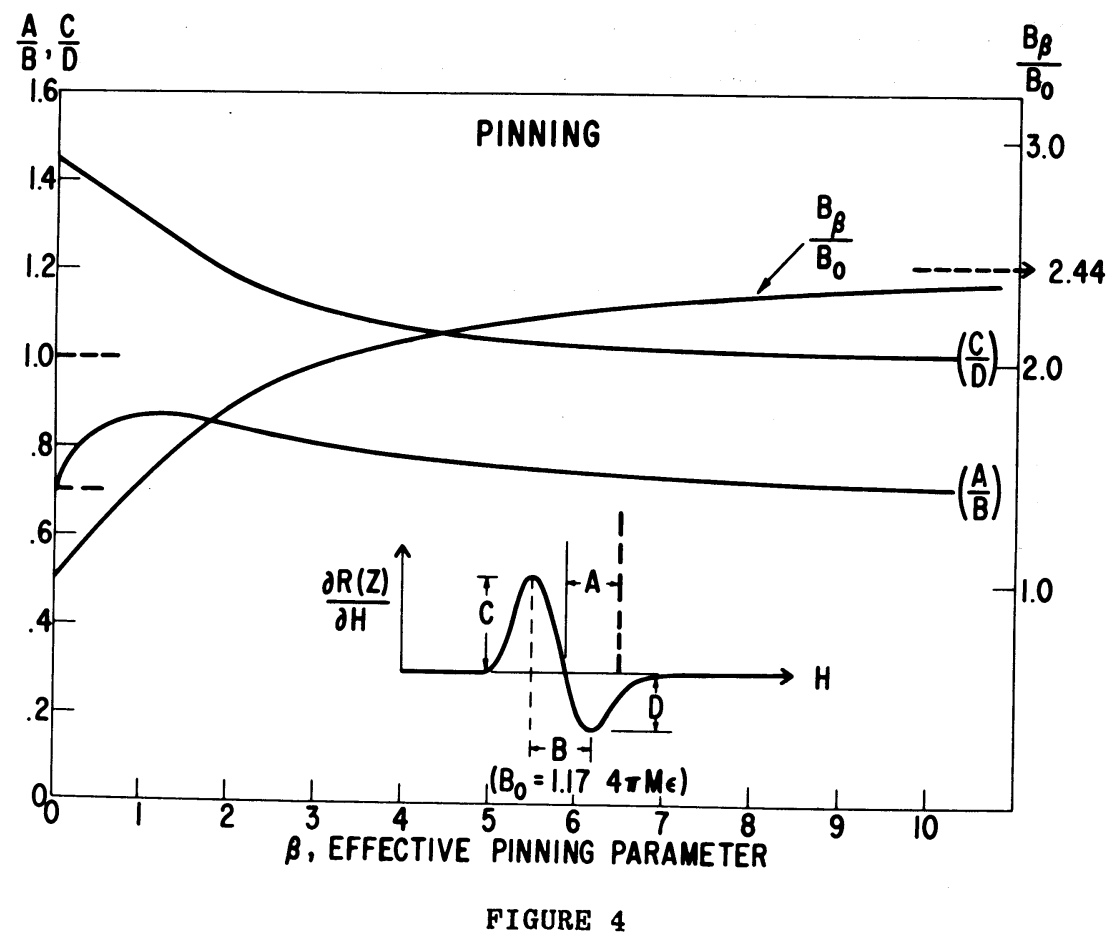

The relative width, asymmetry and shift of the resonance absorption as a function of the strength of the surface pinning, $\beta$, as described in the text.

energy into a conducting ferromagnetic metal. From a simple physical argument it is easy to see the main features of this effect. Consider a metallic sample, of dimensions greater than the penetration depth of the incident high frequency energy, to be excited into ferromagnetic resonance. The resonance excitation is clearly not the uniform precession with all spins parallel since the surface spins are effected by the r.f. fields while the volume spins are not. There must result an angular deviation between neighbouring spins within the skin depth $(\delta)$ and this deviation gives rise to an exchange field $H_{\mathbf{e x}}=A / \bar{M} \delta^{2}$ (where $A$ is the exchange stiffness constant and $M$ the magnetization per unit volume). This field gives rise to a shift in the resonance position to lower values in externally applied fields (at fixed frequency) since it is an addotional restoring force operative on the system. It is also the source of a broadening of the resonance since $\delta \alpha \infty \mu^{-1 / 2}$ i.e. where $\mu$ is the effective permeability. Because $\mu$ itself is a resonant quantity, the penetration 
depth varies as the resonance is traversed and the result is an inhomogeneously broadened resonance i.e. $H_{\text {ex }}$ is not a constant. While earlier workers underestinated this effect at room temperature, the correct estimate of it was first given by Ament and Rado [8](AR). The evaluation of the AR results applied to iron whiskers is shown in Fig. 2. No damping term was used nor did one appear to be needed in that case. The characteristics of the effect are a shift, a breadth and an asymmetry that (without damping) depend on the exchange stiffness, $A$, the magnetization, $M$, and the electrical resistivity, $\rho$, only. When both $M$ and $p$ are assumed to be the same as in bulk iron one may deduce the value of $A$ to be $2.5 \pm .5 \times 10^{-6} \mathrm{erg} / \mathrm{cm}$ for iron (at $25^{\circ} \mathrm{C}$ ). This value compares favourably with others and may be considered a proper independent estimate. What happens in the case of nickel is not so straightforward. After careful selection of a large number of whisker and platelet samples of nickel, the resonance linewidths are found to be much broader and less asymmetric than that predicted for 'exchange only'; e.g. $\Delta H$ ' s typically observed are 130 oersteds to compare with 37 oersteds predicted by 'exhange only' estimates. There are two reasonable possibilities to explore here since we must assume that the crystal perfection of these nickel samples is comparable to that of the iron samples studied previously. (Both the optical appearance and the sharpness of the single crystal diffraction spots support this assumption.) The possibilities for additional line width are (1) surface spin pinning, and (2) an intrinsic damping mechanism of unknown origin. It seems intuitive that the latter mechanism must give additional linewidth; the former mechanism broadens the resonance simply by forcing an increase in the deviations between neighboring spin directions and thus enhancing all of the exchange effects earlier described. We must now face up to the problem of calculating precisely what effect each of these possibilities will contribute and then try to distinguish between them experimentally. While Ament and Rado and Macdonald have set down the equations for exchange effects with damping and Rado and Wertman [20] have considered complete spin pinning; Kaganov and Lu [21] have written down the solutions for arbitrary strength of pinning thus extending the earlier results. In these treatments the damping term takes the Landau-Lifshitz form. We shall use that one here since there are thermodynamic arguments for the correctness, in principle, of a L.L. term - i.e. relaxation towards the instantaneous field [21b]. Furthermore, on the basis of Faraday rotation in $\mathrm{MnF}_{2}$, Portis and Teaney [21c] concluded that a term of this form was needed.

The treatment of Ament and Rado considers the surface impedance that a ferromagnetic metal, in an externally applied d.c. magnetic field, presents to incident high frequency energy. The d.c. and r.f. magnetic fields are in the sample surface and orthogonal to each other. The problem is to solve simultaneously Maxwell's electromagnetic equations with the proper boundary conditions and the dynamical equation of the magnetization; i.e. the Landau-Lifshitz equation. The latter equation is used in the form

$$
\frac{1}{\gamma} \frac{\partial \bar{M}}{\partial t}=\bar{M} \times \bar{H}+\left(\frac{2 A}{M^{2}}\right) \bar{M} \times \nabla^{2} \bar{M}-\left(\frac{\lambda}{\gamma M^{2}}\right) \bar{M} \times[\bar{M} \times \bar{H}]
$$

where the term $\nabla^{2} \bar{M}$ accounts for the exchange torques.

The solution obtains the ratio of the r.f. electric field to the r.f. magnetic field intensity at the sample surface; i.e. the surface impedance. Ament and Rado find it convenient to introduce an 'equivalent isotropic permeability', $\mu_{e}$, which is simply the permeability that will give the same surface impedance as the system considered. Thus the surface impedance becomes $Z_{\text {surf }}=\left(-\mu_{e} \omega / 4 \pi i \sigma\right)^{1 / 3}$; where $-4 \pi i \sigma / \omega$ is the effective dielectric constant that is convenient to use with metals in electromagnetic problems. ( $\sigma$ is the conductivity and $\omega$ the angular frequency of the r.f. fields.) 
The solution for $\mu_{e}$ is given by Ament and Rado (without pinning) as

$$
\mu_{e} \equiv \mu_{1}-i \mu_{2}=\frac{\eta-\Omega^{2}+i \Omega L+2 \varepsilon(1+i)}{\left[\eta-\Omega^{2}+i \Omega L+\varepsilon(1+i)\right]^{2}}
$$

The symbols are defined as

$$
\begin{gathered}
\eta=\frac{H}{4 \pi M}, \quad \Omega=\frac{\omega}{4 \pi M \gamma}, \quad L=\frac{\lambda}{M \gamma}, \varepsilon^{2}=\frac{A}{2 \pi M^{2} \delta^{2}} \\
\text { where } \delta=\left(\frac{\mathbf{c}^{2}}{2 \pi \omega \sigma}\right)^{1 / 2}
\end{gathered}
$$

is the classical skin depth for unit permeability. The solution is valid for $\eta, \Omega^{2}, L^{2}, Q L$, and $\varepsilon<<1$ (numerical solutions are also possible from the 'non-approximate' equations but we shall use the approximate solutions for simplicity, recognizing the limitations of this solution). Kaganov and Lu extended this treatment to include arbitrary surface spin pinning. (Arbitrary in magnitudes but specific in symmetry; namely, in the plane of the sample. Pincus [22] has solved the case of a perpendicular d.c. field with pinning.) The Rado and Weertman general $\partial \bar{m} / \partial n+\alpha \bar{m}=0$ ( $m$ is the r.f. magnetization and $n$ the normal to the surface, $\alpha=K_{s} / A$, the surface anisotropy divided by the exchange stiffness constant). The result obtained by Kaganov and $\mathrm{Lu}$ is given here as

$$
\mu_{e p}=1\left[\frac{(N+1+(\Omega L+2) i)^{1 / 2}+\beta}{\varepsilon+i+\beta[N+1+(\Omega L+2) i]^{k / 2}}\right]^{2}
$$

in which $\varepsilon N=\eta-\Omega^{2}+\varepsilon$ and $\beta=\alpha \delta \sqrt{\varepsilon}$.

It would be possible, within this framework [equation (4)], to solve for line shapes and positions with arbitrary surface anisotropy and arbitrary damping but we believe it more informative to separate them in order to see the major features of each. What we need, to compare to experiment, is the quantity $R\left(Z_{u \times f}\right) \propto\left[\left(\mu_{1}^{2}+\mu_{2}^{2}\right)^{1 / 2}+\mu_{2}\right]^{/ /}$as is readily obtained and since it is experimentally convenient to determine $\partial R\left(Z_{\mathrm{sur} f}\right) / \partial H$ at fixed frequencyk it is this latter form that we have calculated. We consider first the case of no damping $(L=0)$ but with surface spin pinning and calculate (with the aid of an electronic computer) the effects that result as the strength of the pinning is varied. Kaganov and Lu and also Frait [23] have evaluated these effects in an effort to understand observed resonance behaviour thereby; we present our results in a somewhat different form however so that direct comparison to experimental line shapes may be made. In Fig. 3 are displayed a set of 'pinned' resonance absorptions as the derivative, with respect to the magnetic field, against the variable $\varepsilon N \equiv \eta-\Omega^{2}+\varepsilon$. Thus abscissal distances, $\varepsilon \Delta V$, will be measured in $\Delta \eta$ units or $\Delta H=\varepsilon 4 \pi M \Delta N$ defines the scale in field units. The usual resonance, without exchange effects would occur for $\eta=\Omega^{2}$ (i.e. $N=1$ on our plot). To give a feeling for the size of the surface energy (say here for the case of nickel metal at $9 \mathrm{kMc}$ and $25^{\circ} \mathrm{C}$ ) we note that a $\beta=1$ corresponds to a $K_{s} \doteq 1 \mathrm{erg} / \mathrm{cm}^{2}$ (using $A=1 \times 10^{-6} \mathrm{erg} / \mathrm{cm}$ and $\left.\delta=10^{-5} \mathrm{~cm}\right)$. From this figure and others, the determination of line shapes and positions may be made as a function of the strength of the pinning. This is displayed in Fig. 4. As pinning strength is increased the line broadens, shifts, and becomes less asymmetric. There are several things to note. First, pinning alone cannot account for the observed linewidths (if, in fact, it is present at all, - a point we shall pursue later) because for 
complete pinning the linewidth (appropriate to our experiments at $25^{\circ} \mathrm{C}$ ) is only 90 oersteds and would be symmetric. Rado and Weertman noted this symmetry of line for large pinning earlier but did not consider intermediate strength pinning. We see here that a broadened, asymmetric and shifted line could occur with pinning of the sort considered here but if we consider the concomittant asymmetry as locating the appropriate value of $\beta$ then the line breadth would be much smaller than we observe in our experiments. The line shift is here determined to be approximately a constant fraction of the line breadth, a result that seems not unreasonable from the result for exchange effects without pinning. It is not obvious on intuitive grounds or from simple physical arguments why the line becomes symmetric in the limit of immobilised surface spins. It is clear however that we must look elsewhere to understand the source of linewidth in the samples here studied.

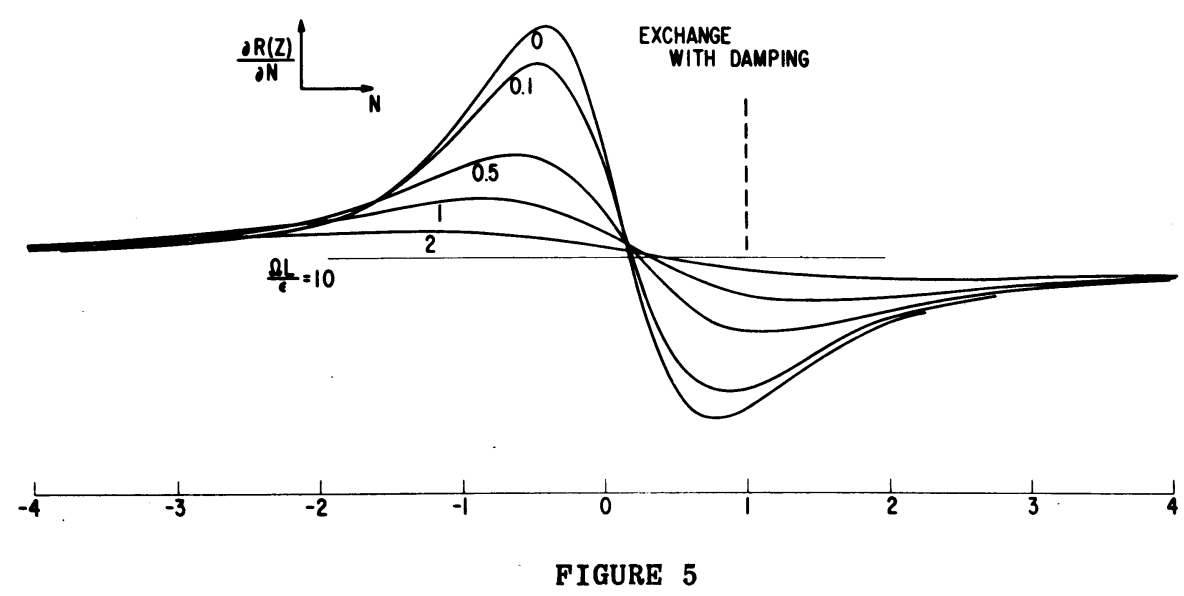

The effect of damping on the resonance absorption line shape. The parameter $\Omega L / \varepsilon$, defined in the text, is the ratio of damping to exchange. The dashed line indicates the location of the resonance in the absence of exchange effects.

Let us now consider the effect of damping in the absence of pinning. We proceed immediately from equation (4) setting $\beta=0$. This equation may be brought to the form

$$
\varepsilon \mu_{e}=\frac{N+1+i(2+\kappa)}{[N+i(1+\kappa)]^{2}} \text { with } \kappa=\frac{\Omega L}{\varepsilon} \text { and again with } \varepsilon N \equiv \eta-\Omega^{2}+\varepsilon
$$

Expression (5) may be used to determine, by numerical evaluation, the absorption line shapes, positions and widths. A set of lines (presented again in derivative form) are given in Fig. 5 with the effective damping to exchange parameter $(\Omega L / \varepsilon)$ a variable. From such evaluations may be determined characteristic line measures and these are shown in Fig. 6. In order to cover the entire range of the damping to exchange ratio we must consider, in addition to the damping as a perturbation on the exchange dominated line, the exchange as a perturbation on the damping dominated line. In that case we use the expression [again derived from (4)]

$$
\begin{aligned}
& \Omega L \mu_{e}=\frac{N^{*}+K^{-1}+i\left(2 K^{-1}+1\right)}{\left[N^{*}+i\left(K^{-1}+1\right)\right]^{2}}, \\
& \text { where } \Omega L N^{*}=\eta-\Omega^{2}+\varepsilon .
\end{aligned}
$$

The result of the evaluation may now be given for $0 \leqslant \Omega L / \varepsilon \leqslant \infty$ by joining the two results 
just described at $\Omega L / \varepsilon=1$ and presenting them in the form of Fig. 7. Thus for any value of damping we have now a means of determining the line width, shape and positional shift. Conversely, for a given shape and width, an estimate of the damping is possible and with it an appropriate shift in the resonance field position. A convenient representation of the line width may be obtained from either Fig. 6 of Fig. 7. For the range $\Omega L / \varepsilon<1$, the linewidth dependence on damping may be accurately described by

$$
\Delta H_{\varepsilon \lambda}=\Delta H_{\varepsilon 0}+0.83_{2} \Delta H_{0 \lambda}
$$

where $\Delta H_{\varepsilon \lambda}$ is the line width due to exchange and damping while $\Delta H_{\varepsilon_{0}}$ is the linewidth to be expected for exchange only (equal to $0.8154 \pi M \varepsilon$ ); $\Delta H_{0 \lambda}$ is the linewidth to be expected for a Landau-Lifshitz damping term, $\lambda$, without other broadening effects present and equals $\frac{3}{2} \frac{\omega \lambda}{\gamma^{2} M}$. (All references to $\Delta H$ here refer to the separation between inflection points of the absorption vs field curve; i.e. the separation between extrema in the derivative presentation).

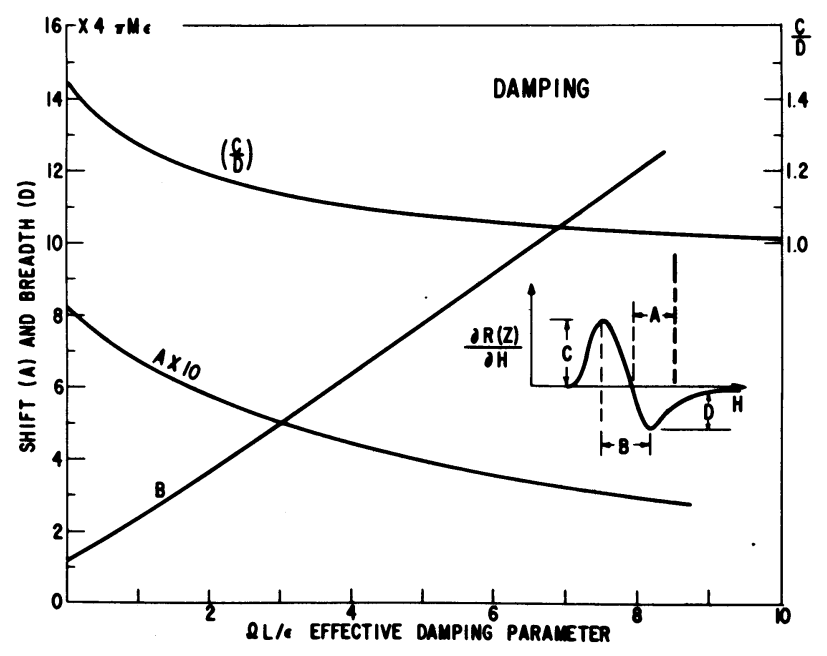

FIGURE 6

The relative width, asymmetry and shift of the resonance absorption as a function of the strength of the damping relative to exchange (1.e. $\Omega L / \varepsilon$ ).

With this part of the analysis done let us turn our attention to the experimental observations and see if, in fact, there is any contact between these and the analytic description given above. In Fig. 8 are displayed a group of experimental results for nickel metal single crystals. Representative plate and whisker data of the field for resonance and line width are shown as a function of temperature at $9 \mathrm{kMc}$. It is our task to understand these data, including the orientation dependence of the field for resonance as well as the line width.

The platelet crystals occur in two morphological types; by far the most abundant are the (100) plane types. The other group grows with (111) crystalographic planes parallel to the growth plane and are found only rarely. The whisker crystals of nickel also have two morphological types, one has the axial orientation colinear with a $<100>$ crystallographic direction; 


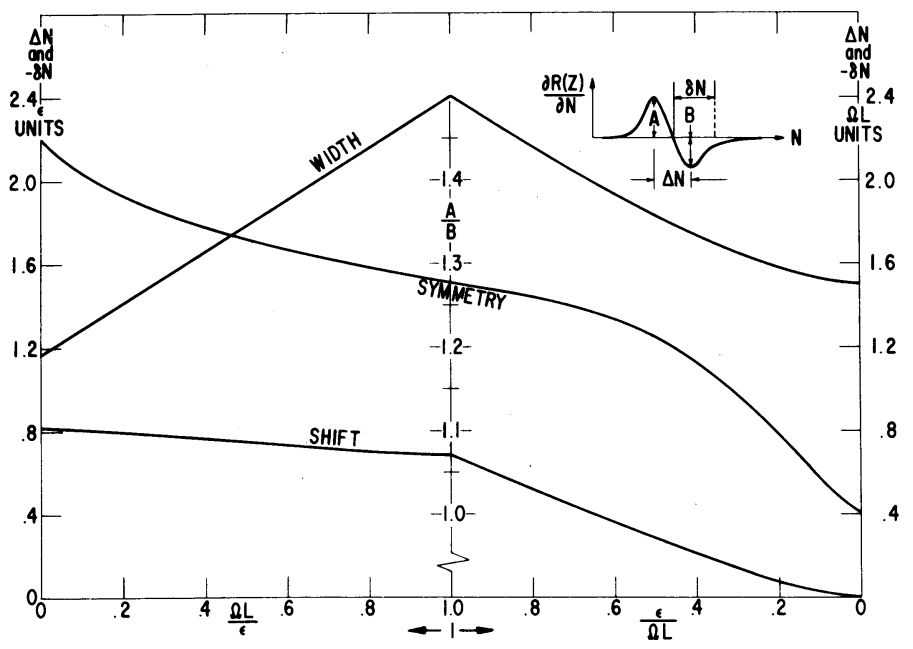

FIGURE 7

The relative width, asymmetry and shift of the resonance absorption covering the entire range of damping. i.e. $0 \leqslant \Omega L / \varepsilon \leqslant \infty$ obtained as described in the text.

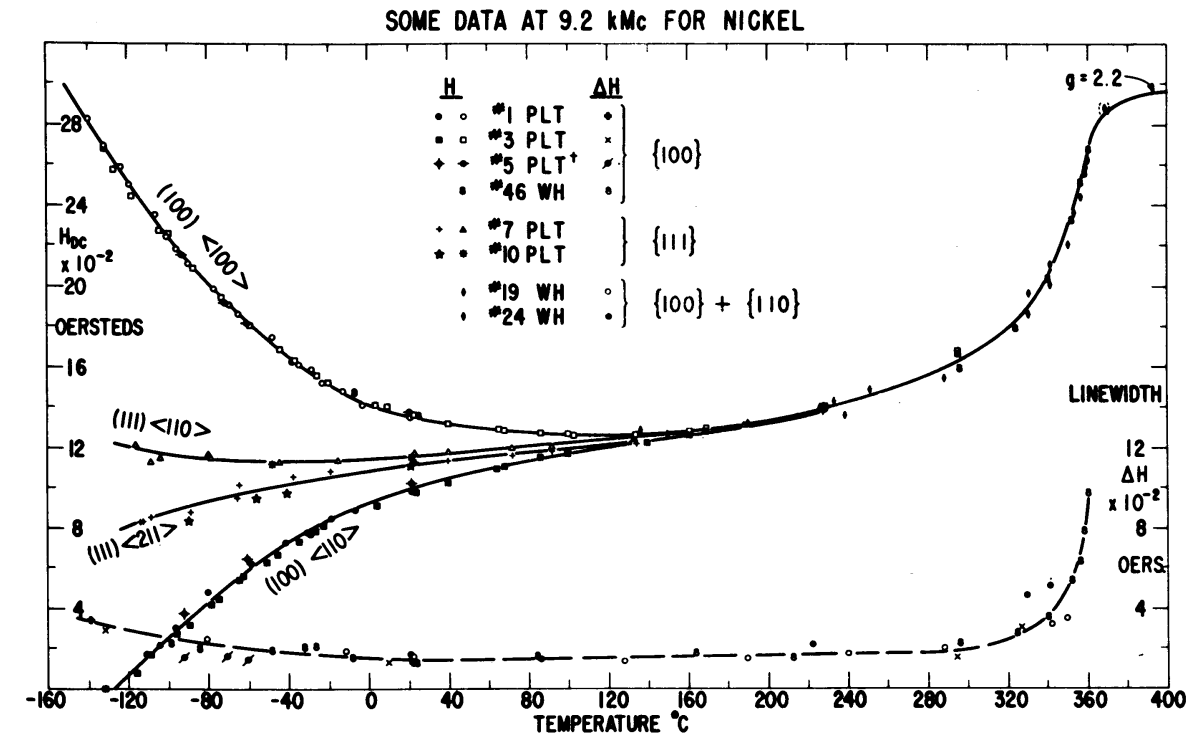

FIGURE 8

\begin{abstract}
Some representative resonance data for nickel. The externally applied field for resonance for several important geometrical arrangements and the line width are
\end{abstract} displayed as a function of temperature through the Curie point $\left(360^{\circ} \mathrm{C}\right)$.

the other with a $<110>$ crystallographic direction parallel to the growth direction. It is easy to use the platelet geometry to obtain data from which the magneto-crystalline anisotropy constants may be deduced and that data is displayed in the figure, the whisker data there presented is selected from higher temperature observations where, because of rapid loss of signal, 
the whisker enhancement [24] feature is useful to pursue the resonance near the Curie temperature $\left(360^{\circ} \mathrm{C}\right)$. Other whisker data will be given and discussed later. For the platelets we show the field for resonance along the principle crystallographic directions contained in the sample planes. These are the appropriate ones for analytic treatment since a principal constraint imposed by experimental geometry confines the magnetization to be in the sample plane. The magnetocrystalline anisotropy in a cubic ferromagnet is usually described in terms of the direction cosines of the magnetization with respect to the cube axes, thus the energy is represented as $E_{k}=K_{0}+K_{1}\left(\alpha_{1}^{2} \alpha_{2}^{2}+\alpha_{2}^{2} \alpha_{3}^{2}+\alpha_{3}^{2} \alpha_{1}^{2}\right)+K_{2}\left(\alpha_{1}^{2} \alpha_{2}^{2} \alpha_{3}^{2}\right)+\ldots$. these are the terms of lowest order that are consistent with cubic symmetry. When the total orientation dependent energy is treated as prescribed by equation (1) the following expressions are found for the principle planes indicated and the directions within those planes.

$$
H \|<100>; \quad\left(\frac{\omega}{\gamma}\right)^{2}=\left[H_{100}+\frac{2 K_{1}}{M}+4 \pi M\right]\left[H_{100}+\frac{2 K_{1}}{M}\right]
$$

(100)

$$
\begin{aligned}
& H \|<110>; \quad\left(\frac{\omega}{\gamma}\right)^{2}=\left[H_{110}+\frac{K_{1}}{M}+\frac{K_{2}}{2 M}+4 \pi M\right]\left[H_{110}-\frac{2 K_{1}}{M}\right] \\
& H \|<110>; \quad\left(\frac{\omega}{\gamma}\right)^{2}=\left[H_{110}^{\prime}-\frac{K_{1}}{M}-\frac{K_{2}}{6 M}+4 \pi M\right]\left[H_{110}^{\prime}+\frac{K_{2}}{3 M}\right]
\end{aligned}
$$

(111)

$$
\begin{aligned}
& H \|<102>; \quad\left(\frac{\omega}{\gamma}\right)^{2}=\left[H_{112}+\frac{9.45}{64} \frac{K_{2}}{M}+4 \pi M\right]\left[H_{112}-\frac{K_{2}}{3 M}\right] \\
& H \|<110>; \quad\left(\frac{\omega}{\gamma}\right)^{2}=\left[H_{110}^{\prime \prime}-\frac{2 K_{1}}{M}+4 \pi M\right]\left[H_{110}^{\prime \prime}+\frac{K_{1}}{M}+\frac{K_{2}}{2 M}\right] \\
& H \|<100>; \quad\left(\frac{\omega}{\gamma}\right)^{2}=\left[H_{100}+\frac{2 K_{1}}{M}+4 \pi M\right]\left[H_{100}+\frac{2 K_{1}}{M}\right] \\
& H \|<111>; \quad\left(\frac{\omega}{\gamma}\right)^{2}=\left[H_{111}-\frac{4}{3} \frac{K_{1}}{M}-\frac{4}{9} \frac{K_{2}}{M}+4 \pi M\right]\left[H_{111}-\frac{4}{3} \frac{K_{1}}{M}-\frac{4}{9} \frac{K_{2}}{M}\right]
\end{aligned}
$$

The experimental information required to solve these expressions for $K_{1} / M, K_{2} / M$ and $g$ (contained in $\gamma$ ) are the frequency, the magnetization and the appropriate shift caused by exchange effects. The last term, the exchange shift, is to be entered in as an additive field to the externally applied d.c. field. While it is not a dominant term it is necessary to know this shift in order to obtain precise deductions of the $g$ factor. For the appropriate exchange shift we must look more carefully at the line width behaviour of our samples in order to decide what the line width is due to and therefore also the proper shift to adopt for the analysis. 
In Fig. 9 are displayed some linewidth data, normalised to room temperature, for several of the samples examined. The linewidth is seen to increase for decreasing temperature as expected on the exchange conductivity basis (notice that No. 5 platelet is an exception to this as will be discussed below). However, instead of the linewidth decreasing for increasing temperature

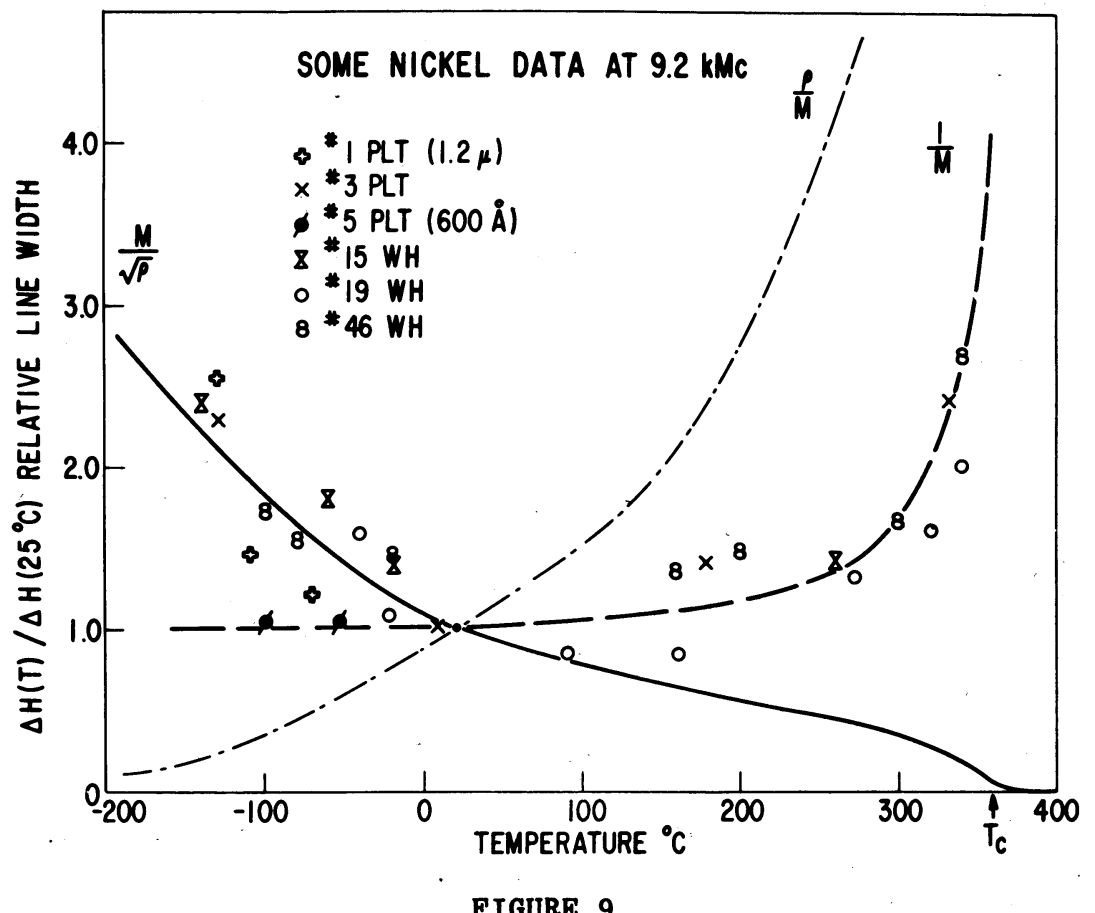

The temperature dependence of the resonance absorption linewidth of several whisker and platelet samples of nickel.

(as the exchange conductivity mechanism predicts) the line width remains essentially constant and then rises rapidly near the Curie temperature. There are several likely mechanisms for line width drawn on the same plot. For exchange and conductivity the monotonic decreasing function (proportional to magnetization/resistivity ${ }^{1 / 2}$ ) is drawn. (The exchange stiffness constant $A$ is taken to be proportional to $M^{2}$.) Linewidth contributions due to surface spin pinning of the sort we have earlier considered would follow this same exchange-conductivity functional dependence. Though we have not yet mentioned the Abrahams-Kittel-Mitchell mechanism [25], such a scheme for line breadth has been proposed and is the result of the spin-flip interaction between conduction electron spins and the resonance excited spin waves.* This mechanism is a true lifetime breadth but is also associated with a shift that, as Hasegawa [26] has pointed out, depends on whether the interaction is adiabatic or isothermal i.e. How does the relaxation time between the conduction electron spin-spin waves compare to the conduction electron-lattice

* There is some question about this mechanism actually being of physical importance since in order for a conduction electron to have a spin-flip interaction it requires an energy comparable to the exchange energy (1.e. the energy difference between $\uparrow$ and $\downarrow$ spin bands) while the energy of a spin wave is proportional to $k^{2}$. For wavelengths comparable to the skin depth, $k$ and therefore the energy are quite small compared to this exchange energy. It is not clear whether the 'screened' exchange value used by Mitchell is correct or not.' 


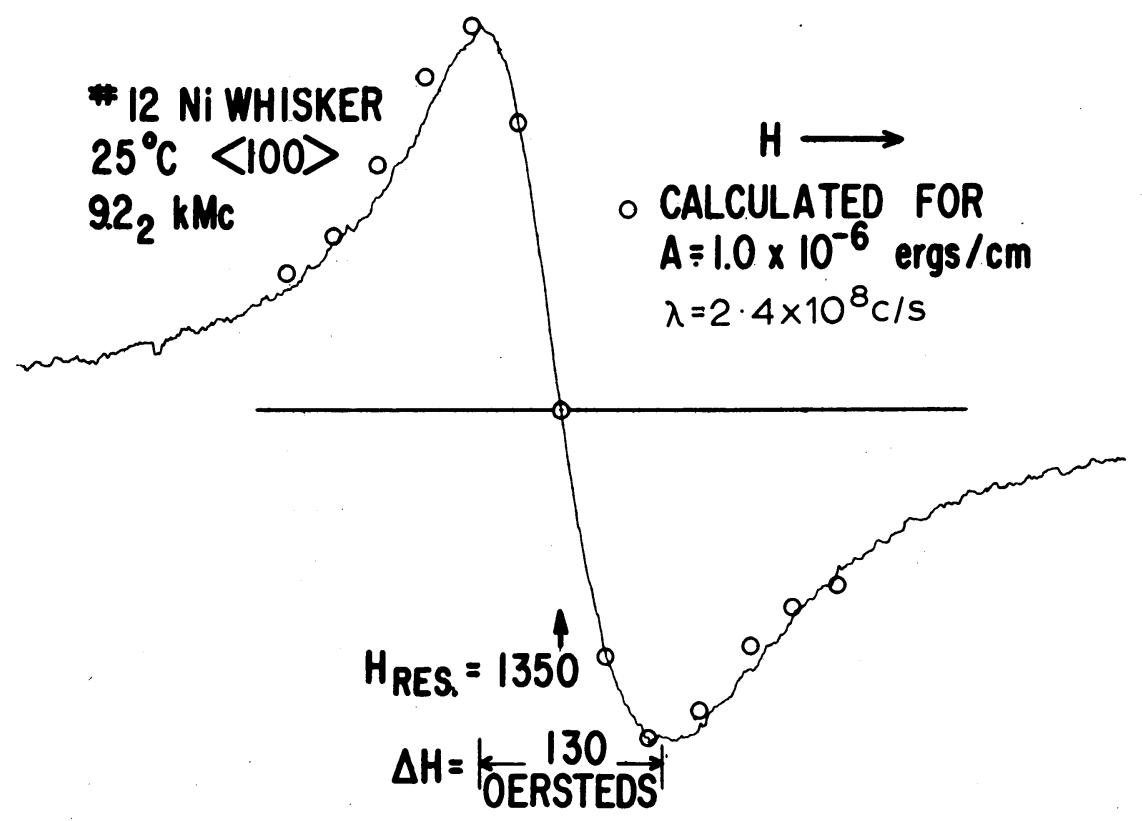

FIGURE 10

Observed and calculated line profiles for a nickel whisker crystal. The display is of the derivative of the real part of the surface impedance with respect to field vs, the applied field. The line width corresponds to a value of $\Omega L / \varepsilon$ that predicts the asymmetry (and the exchange shift) displayed.

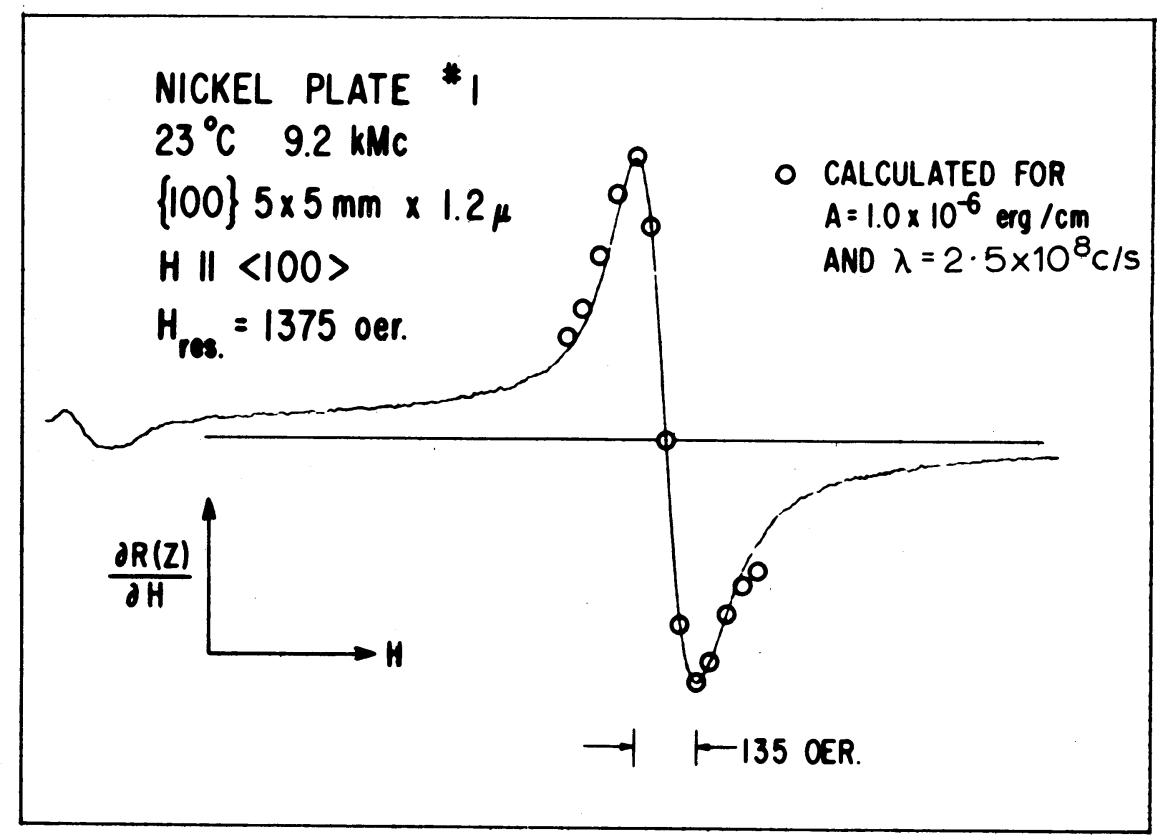

FIGURE 11

Observed and calculated line profiles for a nickel platelet (see Figure 10 caption). 
relaxation time? With simplifying assumptions, the calculation of Mitchell may be brought to the form of the line width being proportional to resistivity/magnetization. This is shown on the normalized plot (Fig. 9) and is seen to be a more rapid rise than the experimental data. The most satisfactory high temperature line width dependence is that predicted by the LandauLifshitz damping term that was earlier discussed. This term contributes a line width that is proportional to the reciprocal magnetization and clearly diverges at the Curie point. Curiously enough it makes a dependence that is quite closely followed by our data when compared as in the figure. The comparison to prediction can be made more acute when we consider the line asymmetry that is calculable for a given width. Suppose we assume that the experimentally observed lines have a breadth due to exchange effects and damping of a Landau-Lifshitz kind. Because our situation is complicated here we cannot make a clearly independent determination of $A$, the exchange stiffness constant, but we shall take a value of $A$ (at $25^{\circ} \mathrm{C}$ ) equal to $1 \times 10^{-6} \mathrm{erg} / \mathrm{cm}$. (The more accurate value of $0.8 \times 10^{-6} \mathrm{erg} / \mathrm{cm}$ will not modify our results greatly for, as we shall see, the system is dominated by damping. Taking a typical experimental result, a $20 \%$ decrease in $A$ increases the deduced value of $\lambda$ by only $3 \%$ ). With the value of $A$ stated above we may now deduce (with our earlier calculations) the value of $\Omega L / \varepsilon$ that will give rise to the observed line width; simultaneously, that same $\Omega L / \varepsilon$ predicts a line asymmetry and shift. In Fig. 10 is shown the observed and calculated line at $25^{\circ} \mathrm{C}$ that results when $\lambda=2.4 \times 10^{8} \mathrm{rad} / \mathrm{s}$ and $A=1 \times 10^{-6}$ $\mathrm{erg} / \mathrm{cm}$ are used for a $<100>$ oriented whisker crystal. Both the line width and asymmetry follow observation quite closely. In Fig. 11 is shown a similar calculation for a nickel platelet where, using the same value of $A$, a value of $\lambda=2.5 \times 10^{8} \mathrm{rad} / \mathrm{s}$ is needed to describe the observed linewidth. (The low field wiggle is probably a domain effect.) The asymmetry of the line is again closely described. These examples are used to show the agreement with theory and in addition are used to indicate that no great difference in line shape is found between the two sample types examined. The unusual behavior of No. 5 platelet, earlier noted for its linewidth vs. temperature dependence (or rather independence) must now be considered. This platelet is somewhat unique in that it is only $800 \AA$ thick. ${ }^{*}$ Because it is so thin the problem of r.f. penetration is much reduced and in fact it is fair to expect that exchange conductivity contributions to the linewidth should be negligible. Thus in the limit of a 'Landau-Lifshitz only' linewidth we would expect an essentially temperature independent linewidth at low temperatures (as the temperature dependence, assuming $\lambda$ constant, is only $M^{-1}$ for this mechanism) and we also expect a symmetrical line shape. In Fig. 12 is shown the observed and calculated absorption for this No. 5 platelet. It is indeed symmetric and requires a $\lambda$ of $2.5 \times 10^{8} \mathrm{rad} / \mathrm{s}$, in reasonable accord with the values needed to understand the behaviour of thicker samples where exchange is also important. It is not clear at this time what the microscopic origin of $\lambda$ might be but it is an observed fact that (evidenced by the increase in linewidth) when the samples are damaged the value of $\lambda$ increases. The collective evidence given above stands strong in favour of using exchange and conductivity effects coupled with Landau-Lifshitz damping and does not associate great importance to surface spin pinning. A final point in support of this position is to consider the frequency dependence of the observed resonance absorption at fixed temperature. The exchange-conduction linewidth (without damping) predicts an $\omega^{1 / 2}$ dependence; the Landau-Lifshitz width goes as $\omega$. Specifically, for the already described type lines, the frequency dependence for a line width arising from both contributions will be found by use of Fig. 6 or by use of equation 7 for small $\Omega L / \varepsilon$. In Fig. 13 are shown the results of calculating absorption lines on the basis of exchange with damping and comparing them to the observations at 9.2 and $34.9 \mathrm{kMc}$ on whisker sample No. 10 at room temperature. The values of $A$ and $\lambda$ are again the same at both frequencies and the same as those used earlier for other samples. The consistency of agreement encountered here between the experiment and our analysis is so striking that we assume that our analysis is based upon a proper physical model and shall adopt it as being so.

- An earlier estimate put this thickness at $600 \AA$. 
Before dismissing surface spin pinning completely, it is worth describing here some experimental efforts to determine the presence of this often invoked possibility. There seem to be three likely pinning mechanisms to consider: (a) Néel type [27] that is due to a difference in symmetry of nearest neighbours about a surface atom compared to a similar atom within the volume; (b) anti-ferromagnetic surface layer coupling to the ferromagnetic surface spins [28]; and (c) dynamic spin pinning [29] due to a lower saturation magnetization of a surface layer and a concomittant detuning of its resonant frequency. If case (a) is operative then a coating

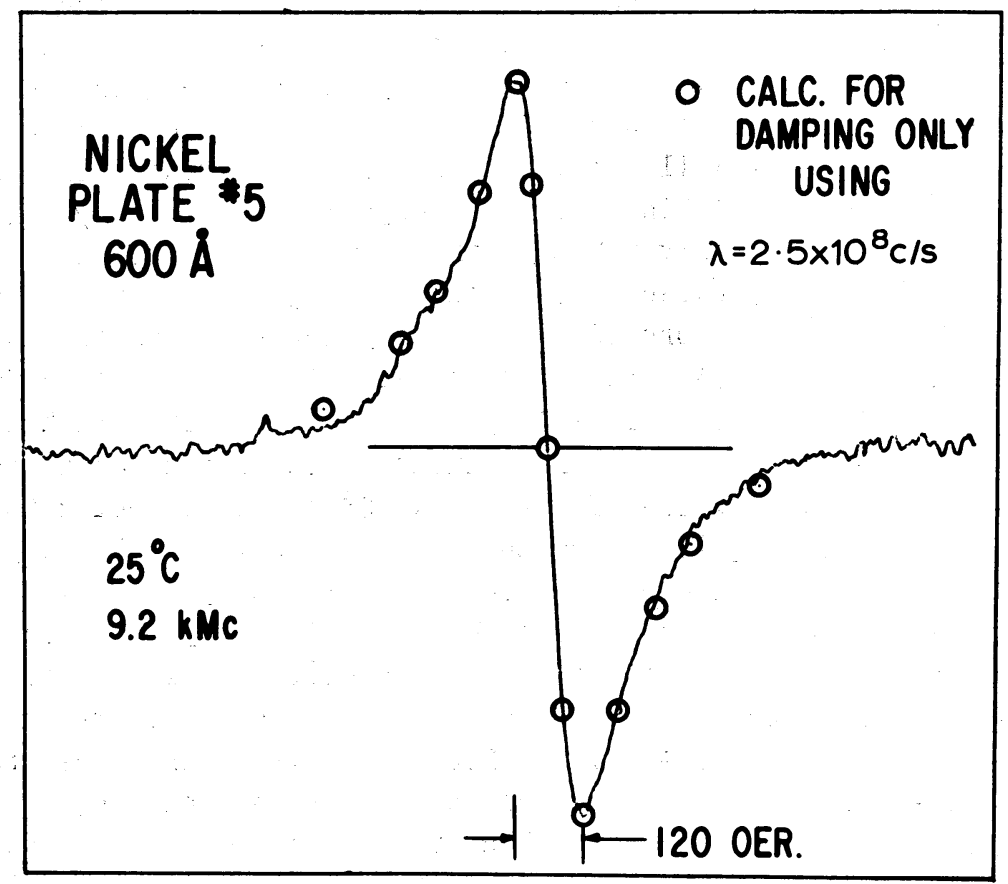

FIGURE 12

The behavior of a $800 \AA$ thin single crystal platelet of nickel. The essentially complete r.f. penetration removes the exchange contribution and the line width and shape may be accounted for with damping only.

(The derivative presentation is again given).

of copper metal onto the surface of the nickel samples should provide the surface atoms with a similar crystalline environment to that experienced by the volume atoms and hence 'unpin' the surface spins. This indicated experiment was performed with negative results; i.e. a whisker with a several hundred angstrom layer of copper (electrolessly deposited) showed the same absorption linewidth as the same whisker prior to deposition of the copper. Case (b) is amenable to examination since the most likely antiferromagnetic surface layer on nickel metal is Nio. The ordering temperature for $\mathrm{NiO}$ is $250^{\circ} \mathrm{C}$ and therefore one might expect that if AFM-FM coupling at the surface is important then as the temperature exceeds $250^{\circ} \mathrm{C}$ and the AFM surface spin ordering vanishes then the linewidth should narrow. This prediction is not observed to occur and as mentioned earlier the line width appears essentially constant from $25^{\circ} \mathrm{C}$ to $300^{\circ} \mathrm{C}$. The mechanism for case (c) is not an effective one for the geometry of our experiments since, a small difference between surface and volume magnetization will 'detune' the resonance only $1 \%$ of the amount that would occur for the perpendicular resonance case for which the mechanism was originally considered. This means that for small deviations in magnetization (as are to be expected in 'good' samples) the detuned resonance for the parallel geometry is still expected to be within the linewidth of the absorption and thus have little pinning effect. This point has 
been demonstrated recently $[30,31]$. Finally, the experimental behaviour of the $800 \AA$ platelet (No. 5) strongly suggests that pinning of any kind is unlikely; at least in these samples and with these geometries. This behaviour is the observation that the field for resonance as a function of temperature and orientation of this $800 \AA$ platelet is essentially the same as that found for much thicker (i.e. $10^{4} \AA$ ) platelets under otherwise identical conditions. If pinning were operative on the surfaces of the $800 \AA$ platelet then its field for resonance should be at least 100 oersteds lower in externally applied field than that required for the thicker samples.

\section{$9.2 \mathrm{kMC}$}
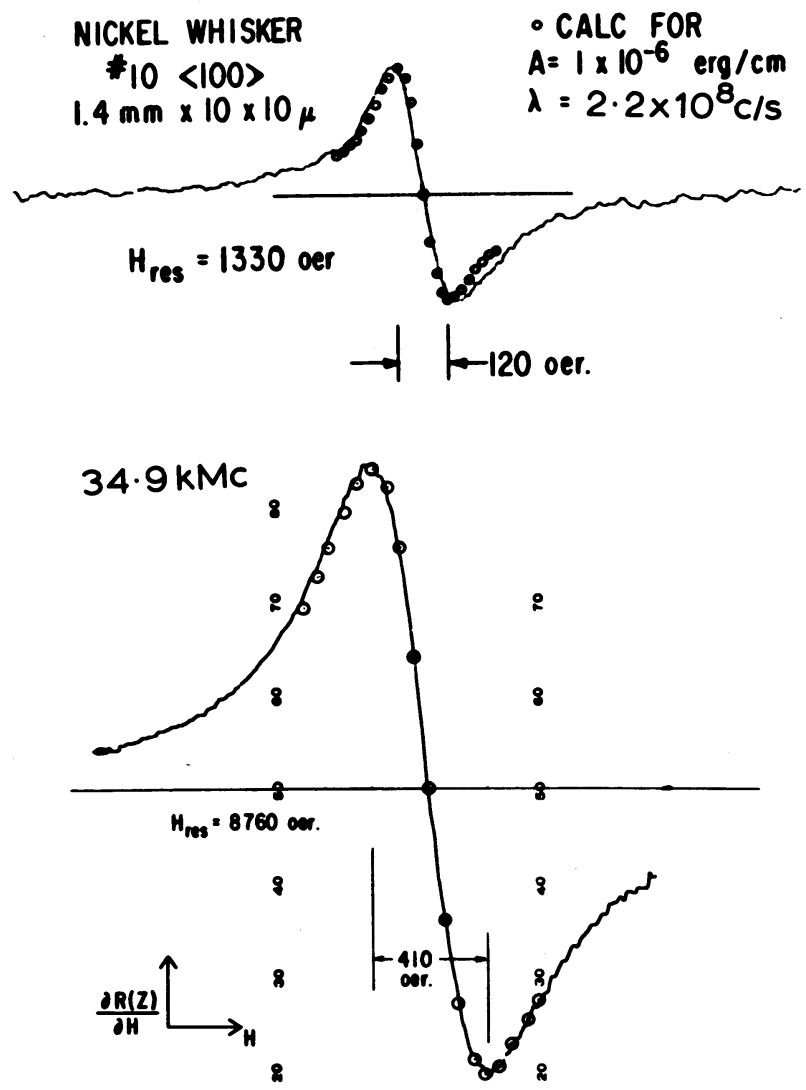

FIGURE 13

The frequency dependence of the resonance absorption calculated on the basis of exchange with Landau-Lifshitz damping. The same whisker at the same temperature is compared to the same theory at two different frequencies (the derivative of the real part of the surface impedance with respect to magnetic field vs. field is shown).

This shift is not found. Our conclusion, from these experiments on nickel metal, casts serious doubt on the presence of appreciable pinning here as we have stated; additionally, the earlier experiments on iron whiskers did not allow appreciable surface pinning to be considered because the line width there observed was essentially accounted for (at the lower temperatures) by the exchange conductivity picture without either pinning or damping. From the recent studies by Wigen, Kooi and Shanabarger [30] and Nisenoff and Terhune [31] on permalloy films, they conclude that surface pinning does not play a measurable role for the 'parallel' orientation but is 


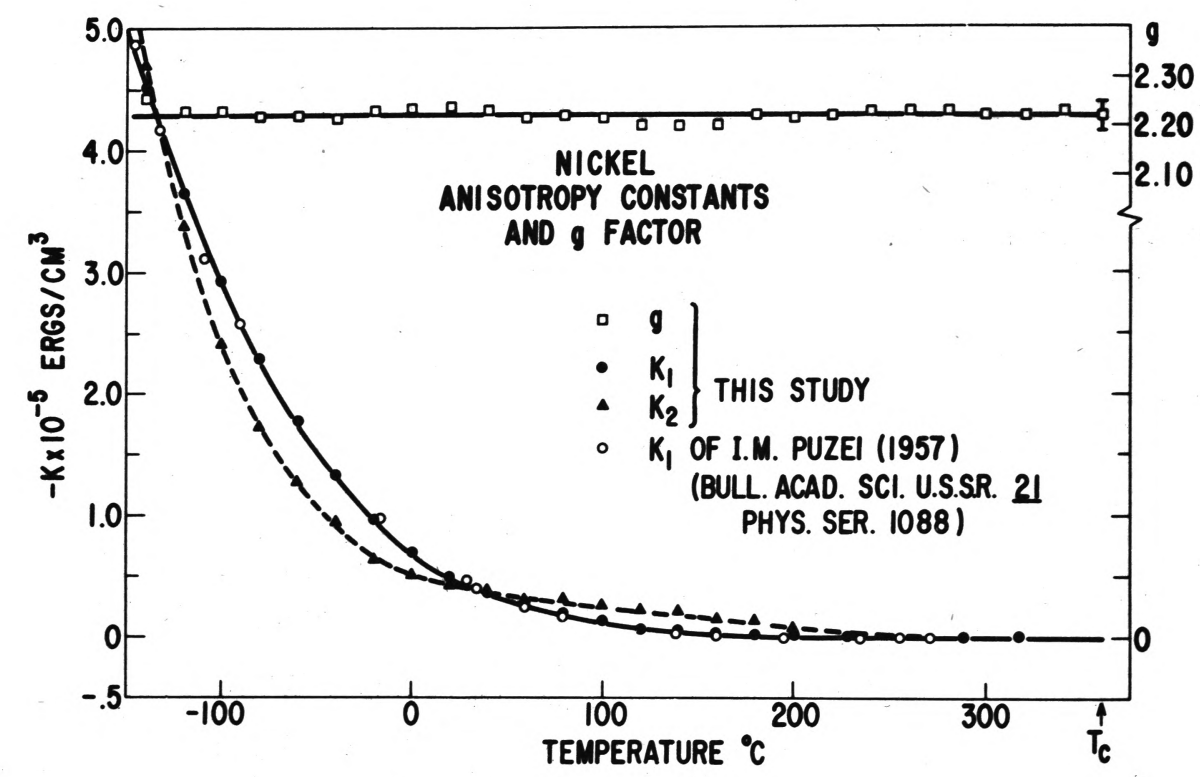

FIGURE 14

The results of the analysis of resonance field vs. temperature given by the simultaneous solution of the appropriate equations (see text).

$K_{1}, K_{2}, g$ are the parameters deduced from this study. $K_{1}$ is compared to results of torque measurements on bulk crystals.

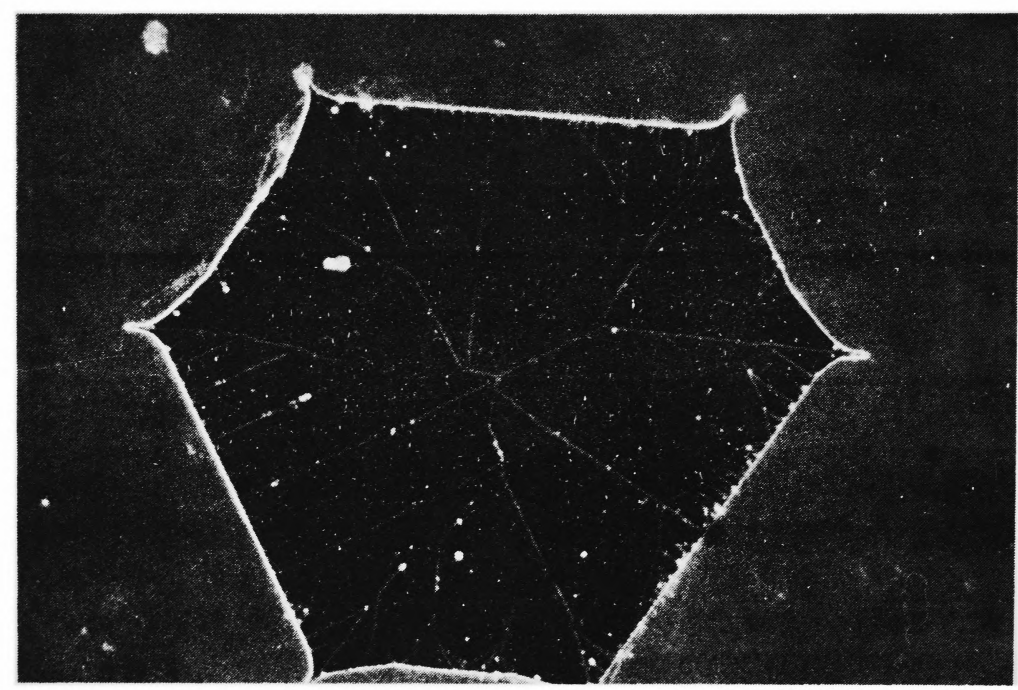

FIGURE 15

Domain pattern of a (111) nickel platelet. The long edges are $\langle 110>$

directions and the fact that the magnetization points perpendicular to these directions is strong. auxiliary, evidence that $K_{2}$ is negative.

required in the 'perpendicular' case. Frait and Mitchell [32] interpret their 'perpendicular' resonance data of permalloy such that a surface anisotropy of about $1 \mathrm{erg} / \mathrm{cm}^{2}$ (easy axis perpendicular to the film surface) is deduced to exist. Thus although surface spin pinning does 
appear necessary for some experimental situations it does not seem to be of importance in the samples or experiments of this study.

The position that we are now in allows us to deduce the temperature dependence of the magnetocrystalline anisotropy constants and $g$ factor from the temperature dependence of the externally applied d.c. magnetic field for resonance (Fig. 8). This determination is made, as earlier outlined, by the simultaneous solution of the appropriate equations (8: a, b, c, d) and with the insertion into the applied field an additional field due to the exchange shift; which field contribution is calculated to be the field shift consistent with the line width and asymmetry detailed above. The effect of this term will be almost entirely on the $g$ factor. Without this correction the $\mathrm{g}$ factor would be slightly larger by a temperature dependent amount (i.e. no correction at $T_{c}$, maximum correction at lowest temperature). For the magnetization of nickel we use the data of Weiss and Forrer [33] determined at the field and temperature of our experiments. The result of the analysis and the solution of these equations yields* the results presented in Fig. 14 while the numerical values are given in Table $I$. The spectroscopic splitting factor, $g$, is found to be $2.22 \pm .03$ and independent of temperature over the range examined; this same $\mathrm{g}$ factor is also found at $35 \mathrm{kMc}$ at $25^{\circ} \mathrm{C}$. The simultaneously determined magnetocrystalline anisotropy constants are both negative over the entire temperature range examined and are very rapidly decreasing functions of the temperature. Thus the $<111>$ crystallographic direction is always the one of lowest energy as far as the magnetocrystalline energy is concerned. In Fig. 14 there are also plotted some determinations of $K_{1}$ made by Puzei [34] using torque techniques on a bulk single crystal. The agreement between that determination and the results of this study are excellent up to $200^{\circ} \mathrm{C}$, where Puzei reports slight positive values for $K_{1}$. Although we do not understand this discrepancy it may be that this result of Puzei's is due to effects of magnetostriction since his measurements are made in very large fields. Aubert [35] has recently made torque determinations of $K_{1}$ and $K_{2}$ on bulk single crystals and finds results in essential agreement with those reported here.

There has been in the past some confusion about the sign of $K_{2}$; whereas Reich [14] and Frait [36] obtained a negative sign for $K_{2}$ using resonance methods, Sato and Chandrasekhar [37] and Birss and Wallis [38], using torque methods, have obtained positive values (although recently these later authors ( $B$ and $W$ ) [38b] have corrected an error in their analysis and find $K_{2}$ to be negative). Hofman, in a recent torque experiment, also finds $K_{2}$ to be negative [39]. As an auxiliary and interesting observation we show in Fig. 15 a domain pattern obtained by Dr. R.W. De Blois on one of the (111) plane platelets as used in this study. The crystallographic orientations are determined by precession camera X-ray techniques which display the reciprocal lattice and from which it is very difficult to misidentify the $\langle 110>$ and $\langle 112>$ directions (as might occur in a back-reflection lane photograph) that 1ie in the (111) crystallographic plane. If $K_{2}$ were positive the $<110>$ directions (parallel to the long edges of the platelet) would be 'easy' directions in the (111) plane and the domain structure would

* The actual method of solution of the equations for $K_{1} / M, K_{2} / M$ and $g$ is done by an iteration process as follows. Equations $8(c)$ and $8(d)$ are solved for $K_{2} / M$ by taking an approximate value for $K_{1} / M$. The value of $K_{2} / M$ thus determined is used in solving $8(a)$ and $8(b)$ for $K_{1} / M$ and $g$. The value of $K_{1} / M$ thus determined is used to reiterate the solution of $K_{2} / M$. This method avoids an otherwise extreme sensitivity of the equations on the magnetization value used, especially of $K_{2} / M$. Equations $8(c)$ and $8(d)$ provide a solution for $K_{2} / M$ that is relatively insensitive to $M$. There will remain a sensitivity of $g$ on the $M$ value used such that a $\Delta M / M$ of $1 \%$ will give a $\Delta g / g$ of about $1 / 2 \%$. There seems to be some evidence (G.Aubert. personal communication and A.J.P. Meyer, personal communication) that the value of $M$ given by Weiss and Forrer may be low by as much as $2 \%$. If so, this would make the g factor deduced here too high by $1 \%$. 
have magnetization oriented parallel to the long edges. The domain pattern seen is quite different from that prediction and is in fact independent evidence that $K_{2}$ is negative; since for $K_{2}$ negative the magnetization should point perpendicular to the $\langle 110\rangle$ edges of the platelet as it is observed to do. It would seem from all of this evidence that the sign of $K_{2}$ is negative and thus settle the apparent discrepancy that has existed in the literature for some time. Among other things, the sign of $K_{2}$ bears upon the sign of the pseudodipolar coupling constant that has been used in some theoretical treatments of anisotropy [40]. The magnitudes of $K_{1}$ and $K_{2}$ also effect this pseudodipolar term and this, in turn, the expected limiting paramagnetic

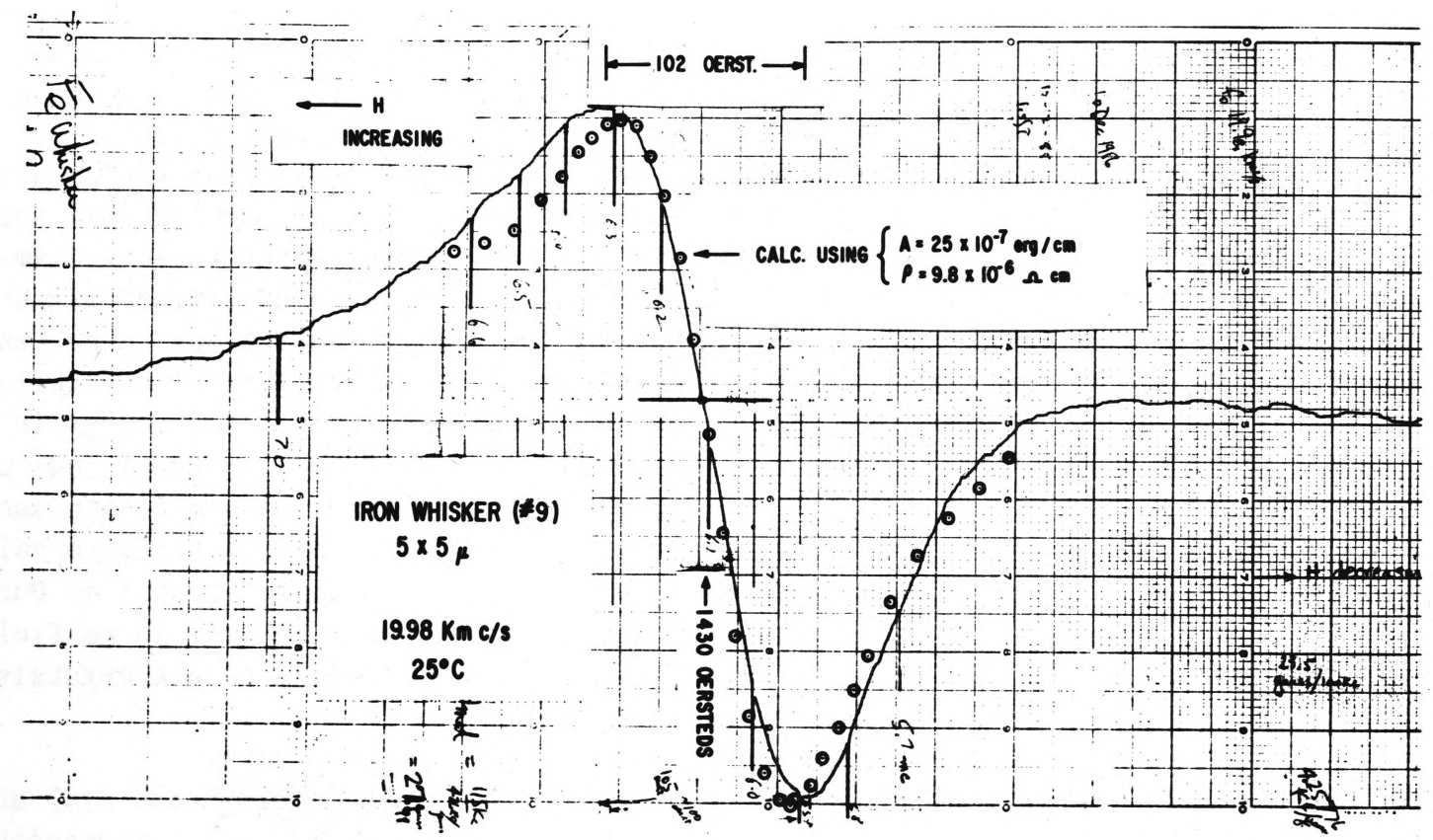

FIGURE 16.

The derivative with respect to magnetic field of the real part of the surface impedance of an iron whisker crystal vs. applied field at $25^{\circ} \mathrm{C}$ and $20 \mathrm{kMc}$. The discrepancy between exchange-only and observation is accountable with a Landau-Lifshitz damping term as in nickel.

resonance line width [41]. The experiments reported here can not give an accurate estimate of this paramagnetic linewidth upper bound since the observed line is not measurable in our apparatus beyond a few degrees above the curie point.

It has also been an attempt of the pseudomultipole approach to account for the implicit relationship between anisotropy constants and magnetization. Since both of the properties are explicit functions of temperature it has been of interest to see if a more direct relationship exists between $K$ and $M$. Theory predicts for systems of cubic symmetry that $\frac{K(T)}{K(O)}=\left[\frac{M(T)}{M(O)}\right]^{n}$ where $n=10$ for $K_{1}$ and 21 for $K_{2}$. This prediction is moderately satisfied in some f.c.c. [42] cobalt studies but does not seem remotely obeyed for nickel metal. If one plots the observations $(K$ vs $M)$, in order to determine the exponent of the power law, then the data of this study yield that for $K_{1}$ the exponent is about 50 while for $K_{2}$ it is about 75 ; these values apply in the range from $-140^{\circ} \mathrm{C}$ to $+20^{\circ} \mathrm{C}$. Our data are not useful below $-140^{\circ} \mathrm{C}$ because of the onset of 
the anomalous skin effect, ${ }^{*}$ however, Puzei [34b] finds from torque determined $K_{1}$ that between 20 and $150^{\circ} \mathrm{K} n \doteq 70$. In terms of the pseudo-multipoles, it would be necessary to use a much higher order of multipoles to get such a dependence as observed here for nickel; it is not clear how to justify this on physical grounds.

\section{Conclusions}

The observations and analysis that have been presented above seem to support the contention that a damping term of the Landau-Lifshitz type does exist in fact. The application of this term with the proper electromagnetic theory seems to account for the temperature and frequency dependence of the resonance absorption linewidth of nickel metal. $\lambda$ itself is taken as a constant with respect to temperature and frequency. It is fair to ask to what extent other materials support this position. In iron metal - again whiskers of high quality - there seems to be supporting evidence. The line shapes calculated at $9 \mathrm{kMc}$ and at $25^{\circ} \mathrm{C}$ are in close accord with exchange-only but the predicted decrease with increasing temperature, expected for exchange-only does not occur and the constant or increasing linewidth variation with temperature is consistent with a $\lambda$ damping term; this term contributing an additional width proportional to $M^{-1}$ as we have already seen. The parameter that describes the effectiveness for additional broadening of the exchange-only linewidth is $\Omega L / \varepsilon$ and this varies as $\omega^{\frac{1}{2}} \lambda / A^{\frac{1}{3}} M$. Thus, if one were to suppose that the $\lambda$ value for nickel is appropriate to iron, then it would make a contribution of

$$
\left(\frac{A_{\text {nickel }}}{A_{\text {iron }}}\right)^{1 / 2}\left(\frac{M_{\text {nickel }}}{M_{\text {iron }}}\right) \doteq\left(\frac{1}{2.5}\right)^{1 / 2}\left(\frac{5}{17}\right) \doteq .21
$$

as large an additional 'exchange-only line width' to the total as for the case of nickel at the same frequency. The discrepancy between observed linewidth and 'exchange-only' in iron metal is shown somewhat more directly in Fig. 15 where the absorption line of another high quality whisker crystal is shown at $20 \mathrm{kMc}$ and at $25^{\circ} \mathrm{C}$. The line was in better accord with the exchangeonly prediction at $9 \mathrm{kMc}$ and $25^{\circ} \mathrm{C}$, but here we see the discrepancy made apparent at the higher frequency. Stated briefly then, a $\lambda$ term of the same size as readily documented for nickel metal in these studies is not inconsistent with earlier observations on iron samples. Because the iron samples' behaviour is actually exhange-dominated, the introduction of a damping term modifies the deduced value of the exchange stiffness significantly. Thus instead of the deduced value $2.5 \pm 0.5 \times 10^{-6} \mathrm{erg} / \mathrm{cm}$ without damping; in the presence of damping (evidenced in Fig. 15 and consistant with $\lambda$ values as in nickel) the deduced value becomes $2.0 \pm 0.5 \times 10^{-6}$ $\mathrm{erg} / \mathrm{cm}$ for iron at $25^{\circ} \mathrm{C}$.

* Anomalous skin effect. When the penetration depth of the microwave energy is comparable to or less than the mean free path of a conduction electron then it is no longer a simple local energy transfer between source and sink. This is a common occurrence in surface impedance studies of metals in general. For non-magnetic metals it is necessary to be at quite low temperatures with quite pure samples to be in the region of this behavior but for ferromagnets the high permeability allows this phenomena to occur at quite modest temperatures. As the permeability depends upon the linewidth, sharper linewidth materials allow higher temperature onsets of this effect. For our samples of nickel and also for iron whiskers there is a distinct change in the character of the resonance (near $-140^{\circ} \mathrm{C}$ for nickel) and the observed line may be interpreted as a mixture of absorption and dispersion just as must result from the onset of the anomalous skin effect. The behavior, although discussed in some detail by Rado [43], does not readily adapt itself to quantitative analysis and we have not attempted to extract $g$ factors and anisotropy constants from the data. 
The conclusions drawn from this study of high quality ferromagnetic metal single crystals are the following:

1) There is evidence that a damping term of the Landau-Lifshitz form actually exists and describes the frequency and temperature dependence of the resonance absorption line width in nickel metal. The microscopic origin of this term is not known.

2) The relaxation frequency, $\lambda$, that characterizes the Landau-Lifshitz term has a value of about $2 \times 10^{8} \mathrm{rad} / \mathrm{s}$ but appears to be structure sensitive.

3) Analysis of the data of nickel metal yields this relaxation term and with it, using an exchange stiffness of $1 \times 10^{-6} \mathrm{erg} / \mathrm{cm}$ at $25^{\circ} \mathrm{C}$, a temperature and frequency independent $\mathrm{g}$ factor equal to $2.22 \pm .03$.

4) The magnetocrystalline anisotropy constants are simultaneously determined in this study. Both $K_{1}$ and $K_{2}$ are found to be negative over the entire temperature range examined, and both have very rapidly decreasing magnitudes with increasing temperature.

\section{Acknowledgement:}

I thank R.W. De Blois for his skilled supply of the samples of this study, for his patient, numerous, assistances and for informative discussions. G. Mahan and T. Moore helped me materially in the use of a GE 225 computer that was employed in much of the data analysis. I am indebted to Professor B. Bleaney and the staff of the Clarendon Laboratory for their hospitality during the writing of this paper and to the John Simon Guggenheim Memorial Foundation for their generosity.

\section{APPENDIX :}

\section{Some experiments with $<110>$ whiskers}

It is noteworthy that $<110>$ axis whiskers with rectangular cross sections have both (110) and (100) facets whereas the $\langle 100>$ axis whiskers of rectangular cross section have only one type of facet, usually (100). For the case of $\langle 110>$ axis whiskers of rectangular cross section one can readily see from equations (8b) and (8e) that the (100) facets should resonate at different external field than the (110) facets - if there were no interaction between facets. Experimentally, as shown in Fig. (A1), these whiskers exhibit one mode only and that occurs intermediate with respect to the fields expected for the individual facet modes. This result suggests that the individual facets are quite tightly coupled, perhaps by dipolar fields, and behave as a unit with the average stiffness of the pair. The absorption line widths for such resonances and not appreciably different from the simpler $<100>$ axis whiskers or the platelet samples. Figure (A1) shows a resonance line for No. 24 sample. The behaviour is satisfactorily analysed just as in the $\langle 100\rangle$ whisker types, using exchange with damping. In Fig. (A2) are shown the results for several different $<110>$ samples and the actual resonant field position seems clearly weighted in proportion to the relative facial area contribution i.e. the more the $(100) /(110)$ area ratio, the nearer to $(100)$ will be the resonance field.

As another interesting experiment of this study let us look at some observations that enable the magnetostriction constant of nickel to be estimated. This experiment was performed on a 
$<110>$ axis whisker but it is not unique to that morphological type sample. In the resonance examination of a whisker it is convenient to mount it inside of a quartz capillary tube and then to mount the capillary into the microwave measuring equipment. A whisker in such a capillary is normally not cemented but allowed to be free to expand or contract during thermal cycling. Figure (A3) illustrates the difference in behaviour of a $<110>$ axis whisker sample when free (a) and then when cemented in place along its entire length (b). Because the quartz

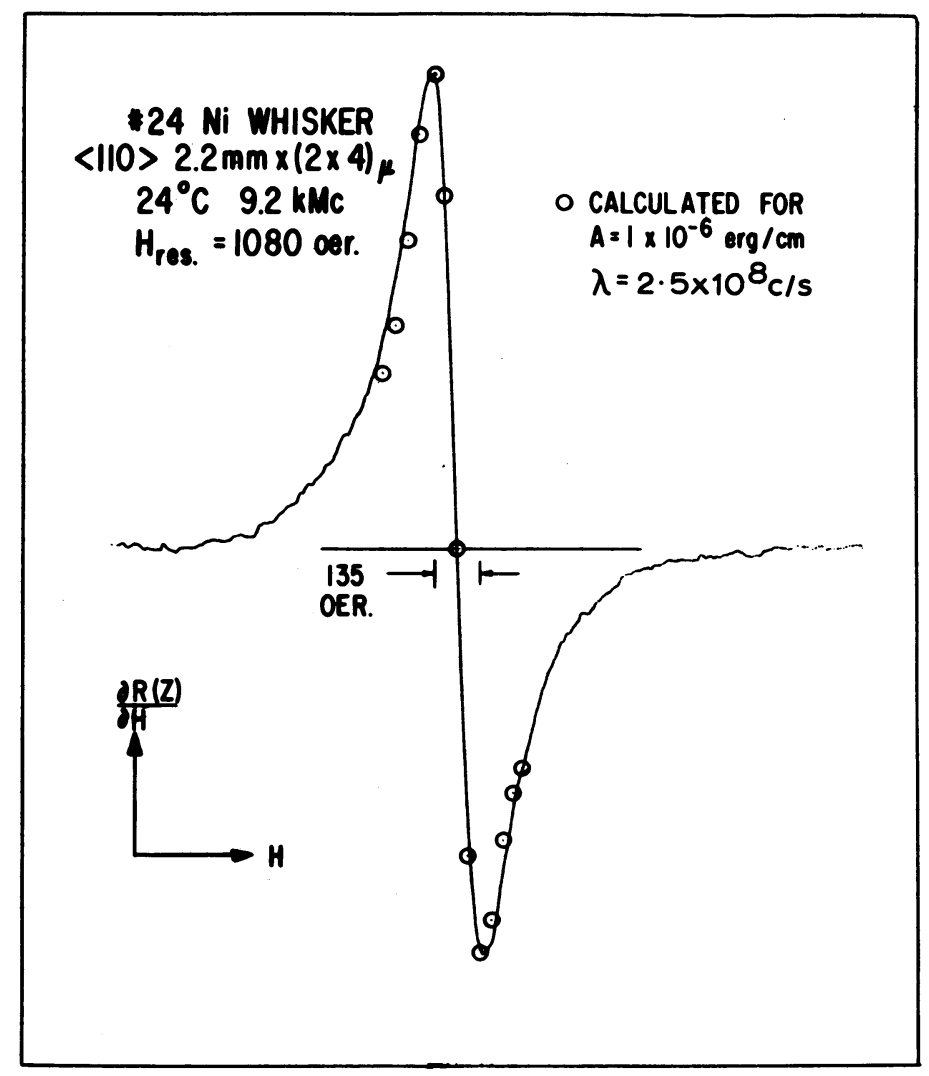

FIGURE A1

Resonance lineshapes of a $<110>$ type whisker crystal. It behaves just as the $<100>$ type samples as far as line shape is concerned and has been analysed in the same way.

capillary has essentially no extension or contraction the whisker, when bonded to the capillary, is under a large effective tension when cooled below the bonding temperature (e.g. $25^{\circ} \mathrm{C}$ ). By considering that the differential thermal contraction on cooling represents an energy $3 / 2 \Lambda s$ and that this energy is equivalent to an effective axial field times the magnetization one obtains $\Lambda=\frac{d H}{d T} \frac{M}{3 Y b}$, where $\Lambda$ is the magnetostriction constant, $d H$ is the change in resonance field between free and cemented conditions that is observed for a temperature change $d T$. $M$ is the magnetization, $Y$ the Young's modulus and $b$ the difference in thermal expansion between quartz and nickel. This analysis leads to $\Lambda_{110}=-26 \times 10^{-6} \mathrm{~cm} / \mathrm{cm}$ for the experiment shown in the figure. When the cement was removed and the experiment repeated the original 'free' data were repeated. This value of $\Lambda_{110}$ is in close accord with values quoted for bulk nickel. 


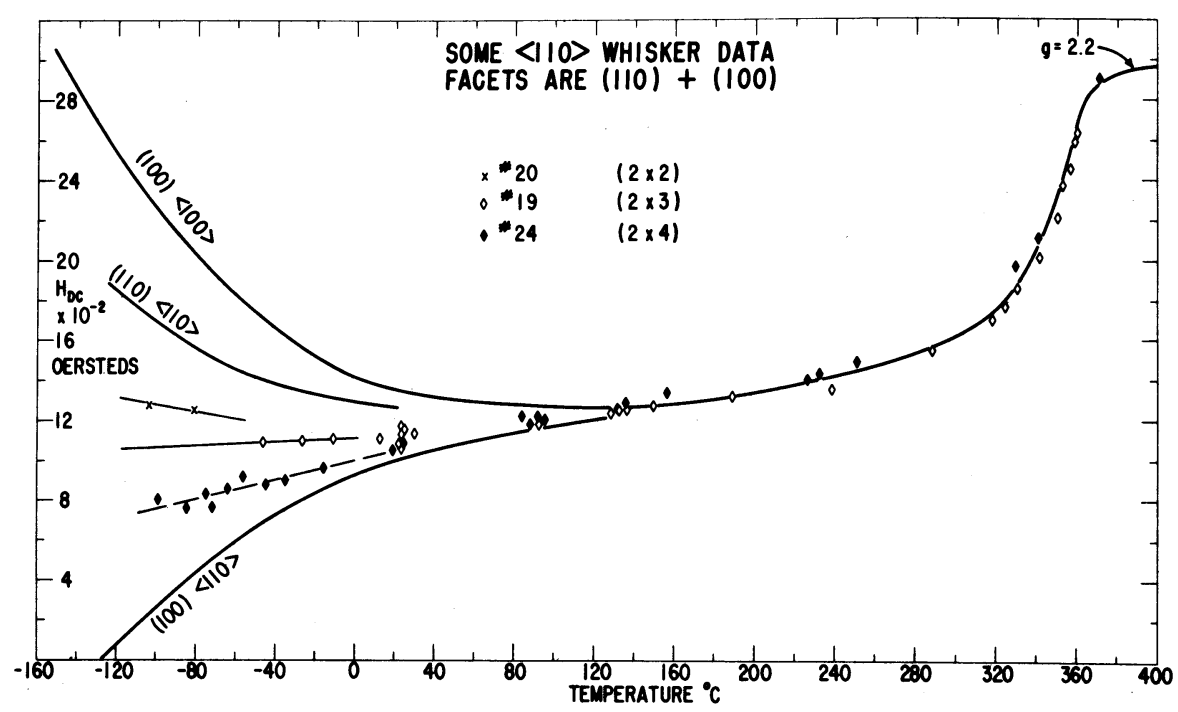

FIGURE A2

The temperature dependence of the resonance position of some $\langle 110\rangle$ whiskers showing that the resonance field depends on the relative facet areas of a given sample - i.e. the larger the ratio $(100) /(110)$, the closer to the $(100)<110>$ prediction is the resonance.

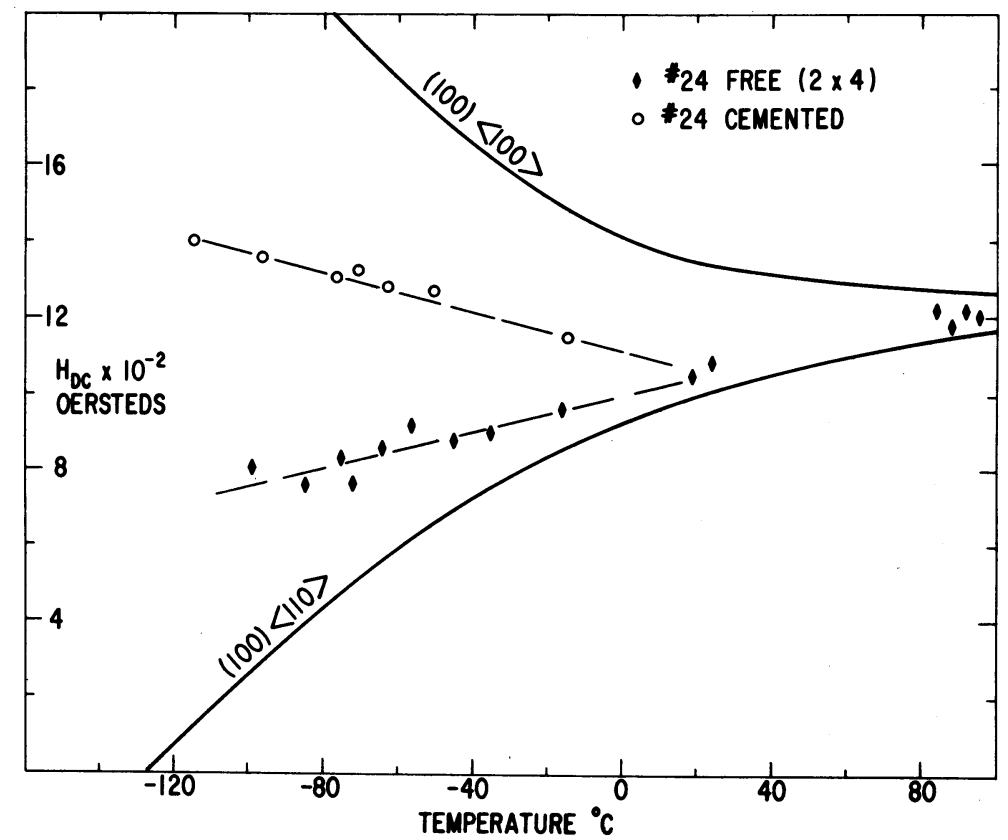

FIGURE A3

The dependence of the field for resonance of a 'free' whisker and the same whisker's dependence when subject to an axial stress. The analysis deduces a value of $\Lambda_{110}$ from these observations that is in excellent agreement with values quoted for bulk nickel. 
TABLE I

The deduced values of $g, K_{1} / M$ and $K_{2} / M$ from this experiment.

The value of $M$ is taken from Weiss and Forrer (see references) at the field and temperature of the experiment; the frequency was $9.22 \mathrm{kMc}$ at $20^{\circ} \mathrm{C}$.

\begin{tabular}{|c|c|c|c|c|}
\hline$T^{\circ} \mathrm{C}$ & $\begin{array}{r}g \\
\pm .03\end{array}$ & $\begin{array}{c}-K_{1} / M \\
\text { oersteds } \\
\quad \pm 5\end{array}$ & $\begin{array}{l}-K_{2} / M \\
\text { oerteds } \\
\quad \pm 10\end{array}$ & $\begin{array}{c}M \\
\text { emu } / \mathrm{cc}\end{array}$ \\
\hline-140 & 2.26 & 897 & 932 & 503 \\
\hline-120 & 2.23 & 728 & 675 & 502 \\
\hline-100 & 2.23 & 586 & 483 & 499 \\
\hline-80 & 2.22 & 462 & 349 & 496 \\
\hline-60 & 2.22 & 358 & 256 & 495 \\
\hline-40 & 2.22 & 270 & 189 & 493 \\
\hline-20 & 2.23 & 197 & 129 & 489 \\
\hline 0 & 2.24 & 142 & 104 & 483 \\
\hline 20 & 2.24 & 103 & 87 & 478 \\
\hline 40 & 2.23 & 78 & 78 & 474 \\
\hline 60 & 2.22 & 58 & 61 & 469 \\
\hline 80 & 2.22 & 40 & 70 & 463 \\
\hline 100 & 2.20 & 28 & 56 & 458 \\
\hline 120 & 2.20 & 18 & 49 & 454 \\
\hline 140 & 2.20 & 12 & 48 & 445 \\
\hline 160 & 2.20 & 7 & 36 & 431 \\
\hline 180 & 2.22 & 5 & 34 & 410 \\
\hline 200 & 2.22 & 7 & 18 & 401 \\
\hline 250 & 2.22 & $0^{+}$ & $0^{+}$ & 365 \\
\hline 360 & 2.23 & 0 & 0 & 44.5 \\
\hline
\end{tabular}

\section{REFERENCES}

1. L. LANDAU and E. LIFSHITZ, Physik, Z. Sowjetunion 8, 153 (1935).

2. J.H.E. GRIFFITHS, Nature 158, 670 (1946).

3. C. KITTEL, Phys. Rev. 76, 743 (1949).

4. J.H. VAN VLECK, Phys. Rev. 78, 266 (1950).

5. H. SUHL, Phys. Rev. 97, 555 (1955). 
6. J. SMIT and H.G. BELJERS, Phillips Res. Reports 10, 113 (1955).

7. C. HERR ING and C. KITTEL, Phys. Rev. 77, 725 (1950) and 81, 869 (1950).

8. W.S. AMENT and G.T. RADO, Phys. Rev. 97, 1558 (1955).

9. J.R. MACDONALD, Phys. Rev. 103, 280 (1956) and Proc. Phys. Soc. (Liondon) A64, 968 (1951).

10. A.M. ClOGSTON, H. SUHL, L.R. WALKER and P.W. ANDERSON, J. Phys. Chem. Solids 1, 129 (1956).

11. L. R. WALKER, Phys. Rev. 105, 390 (1957).

12. J.R. ESHBACH and R.W. DAMON, Phys. Rev. 118, 1208 (1960); J. Appl. Phys. 31, 1045 (1960).

13. N. BLOEMBERgen, Phys. Rev. 78, 572 (1950).

14. K.H. REICH, Phys. Rev. 101, 1647 (1956).

15. Z. FRAIT, (U.S. Mag. Conf. 1964 - At. City, N.J.).

16. D.M. S. BAgguleY, Proc. Roy. Soc. (London) A228, 549 (1955).

17. A.F. KIP and R.D. ARNOLD Phys. Rev. 75, 1556 (1949).

18. D.S. RODBELL, J. Appl. Phys. 30, $187 \mathrm{~S}$ (1959) and in Growth and Perfection of Crystals, edited by Doremus, Roberts and Turnbull, John Wiley and Sons, Inc. N.Y., p. 247 (1958).

19. Z. FRAIT, Czech. J. Phys. 10, 346 (1960) and D.S. RODBELL, unpublished work.

20. G. T. RADO and J.R. WEERTMAN, Phys. Rev. 94, 1386 (1954).

21a. M. KAGANOV and J. LU, Izv. Akad. Nauk SSSR, Phys. Ser. 25, 1375 (1961).

21b. R.K. WANGSNESS, Phys. Rev. 111, 813 (1958)'.

21c. A.M. PORTIS and D.T. TEANEY, Phys. Rev.116, 838 (1959).

22. P. PINCUS, Phys. Rev. 118, 658 (1960).

23. Z. FRAIT, Czech. J. Phys, B13, 535 (1963).

24. D.S. RODBELl, J. Appl. Phys. 30, 1845 (1959).

25. A.H. MITCHELL, Phys, Rev. 105, 1439 (1957) and references contained therein.

26. H. HASEGAWA, Advances in Theor. Phys. 21, 483 (1959).

27. L. NÉEL, J. Phys, Radium 15, 225 (1954).

28. W. H. MEIKLEJOHN and C.P. BEAN, Phys. Rev.102, 1413 (1956).

29. P.E. WIGEN, C.F. KOOI, M.R. SHANABARGER and T.D. ROSSING, Phys. Rev, Letters 9, 206 (1962).

30. C.F. KOOI, P.E. WIGEN and M.R. SHANABARGER, Proceedings of the Internationao Conference on Magnetism (Nottingham) 1964, to appear. 
31. M. NISENOFF and R.W. TERHUNE - to appear.

32. Z. FRAIT and E.N. MITCHELL - Proceedings of the International Conference on Magnetism (Nottingham 1964) - to appear.

33. P. WEISS and R. FORRER, Ann. Phys. Paris. 5, 153 (1926) and 12, 279 (1929).

34a. I.M. PUZEI, Bull. Acad. Sci. USSR, 21, 1088 (orig.) 1077 (trans.) (1957).

34b. I.M. PUZEI, Fiz. metal metalloved 16, 29 (1963).

35. G. AUBERT - personal communication.

36. Z. FRAIT - personal communication.

37. H. SATO and B. ChandRasekhaR, J. Phys. Chem. Solids 1, 228 (1957).

38a. R.R. BIRSS and P.M. WALLIS, J. Sci. Instr. 40, 551 (1963).

38b. R.R. BIRSS and P.M. WALLIS - Proceedings of the International Conference on Magnetism (Nottingham 1964) - to appear.

39. U. HOFMAN, Phys. Stat. Sol. 7, K145 (1964).

40. R.J. JOENK, Phys. Rev. 130, 932 (1963).

41. B. R. COOPER and F. KEFFER, Phys. Rev. 125, 896 (1962).

42. D.S. RODBELL, J. Phys. Sco. Jap. (Suppl. B-1), 17, 313 (1962).

43. G. T. RADO, J. Appl. Phys. 29, 330 (1958).

44. P.E. SEIDEN, Phys. Rev. Letters 14, 370 (1965) suggests that fluctuations in pseudodipolar fields may account for this linewidth by a two magnon scattering process (see reference 10). There is, however, some question about the frequency dependence of that potential contribution as seiden recognizes. 